\title{
Triggers over XML Views of Relational Data
}

\author{
Feng Shao \\ Antal Novak Jayavel Shanmugasundaram \\ Cornell University \\ \{fshao,afn, jai $\} @$ cs.cornell.edu
}

\begin{abstract}
Current systems that publish relational data as XML views are passive in the sense that they can only respond to user-initiated queries over the XML views. In this paper, we propose an active system whereby users can place triggers on (unmaterialized) XML views of relational data. In this architecture, we present scalable and efficient techniques for processing triggers over XML views by leveraging existing support for SQL triggers in commercial relational databases. We have implemented our proposed techniques in the context of the Quark system built on top of IBM DB2. Our performance results indicate that our proposed techniques are a feasible approach to supporting triggers over XML views of relational data.
\end{abstract}

\section{Introduction}

XML has emerged as a dominant standard for information exchange on the Internet. However, a large fraction of data continues to be stored in relational databases. Consequently, there has been a lot of interest in publishing relational data as XML. A powerful and flexible way to achieve this goal is to create XML views of relational data $[11,20,23,29]$. In this way, the data can continue to reside in relational databases, while Internet applications can access the same data in XML format through the XML view. This architecture is shown in Figure 1. As a concrete example, consider a supplier that stores its product catalog information in a relational database. In order to expose the product catalog as an XML web service to buyers, the supplier can create an XML view of the product catalog and expose this view as a web service.

Current systems that support XML views of relational data are passive in the sense that they can only support userinitiated queries over the views. For instance, in the web services example above, current systems only allow buyers to explicitly initiate a request to query the catalog for products of interest. In this paper, we propose an active system that allows users to specify triggers over XML views. Thus,

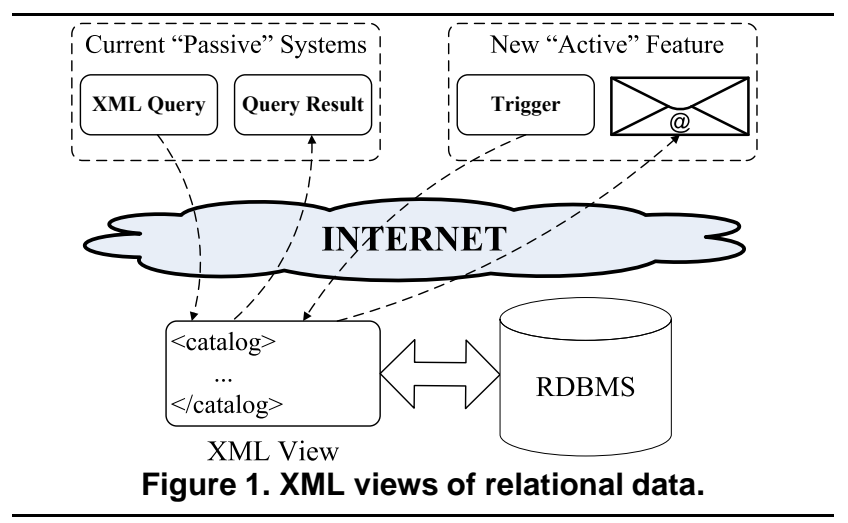

a buyer can set a trigger to be notified whenever a new product is introduced, or when a product of interest goes out of stock, without having to repeatedly query the XML view to detect these changes.

At a high level, there are two approaches to supporting triggers over XML views. The first approach is to materialize the entire XML view, store it in an XML database, and implement XML triggers in this database. However, this approach suffers from the overhead of replicating and incrementally maintaining the materialized XML view on every relational update that affects the view, even though users may only be interested in relatively rare events. Another practical downside of this approach is that it requires a fullfunction XML database that supports incremental updates and triggers, even though the underlying relational database supports all of this functionality and is typically much more optimized for these tasks. Therefore, in this paper, we propose the alternative approach of translating XML triggers into $S Q L$ triggers over the relational data. The primary benefits of this approach are that it fully leverages sophisticated relational technology, does not require an XML database, and avoids having to materialize the XML view.

The main technical contribution of this paper is a systematic way to translate triggers over XML views of relational data into SQL triggers. This translation is fairly challenging because XML triggers can be specified over complex nested XML views with nested predicates, while SQL triggers can only be specified over flat relational tables. Consequently, even identifying the parts of an XML view that could have 
changed due to a (possibly deeply nested) SQL update is a non-trivial task, as is the problem of computing the old and new values of an updated fragment of the view. Another issue is that current commercial relational databases are not very scalable with respect to the number of SQL triggers even though we expect a large number of XML triggers to be specified over XML views exposed as web services.

In this paper, we address the above challenges. Specifically, our two main contributions are: (1) a system architecture for supporting triggers over XML views of relational data (Section 3), and (2) an algorithm for identifying and computing changes in an XML view based on possibly deeply nested relational updates (Section 4). We also show how prior work on scalable trigger processing [14, 5] can be adapted for the XML view problem (Section 5).

We have implemented our proposed techniques in the context of the Quark system built over IBM DB2. One of the original goals of Quark (like XPERANTO [23] and SilkRoute [11]) was to support queries over XML views of relational data. By integrating with Quark, we were able to leverage many of the techniques originally developed for querying XML views, and adapt them to the trigger problem. This suggests that our techniques can be easily integrated into systems that already support queries over XML views of relational data (including relational database systems with built-in XML publishing support). Our performance results using our prototype show that our proposed techniques provide an efficient and scalable way to support triggers over XML views of relational data.

While our focus is on triggers over XML views, our techniques also apply to the less general problem of triggers over (flat) relational views. We note that current relational systems only support INSTEAD OF triggers [24] over unmaterialized views. Using INSTEAD OF triggers, users can manually specify how updates on a view are to be translated into updates on base tables. In contrast, we are solving the problem of automatically inferring when updates on base tables cause triggers on a view to be fired. We are not aware of any published work or commercial systems that support such SQL triggers over unmaterialized views.

\section{Background}

We have developed our trigger processing techniques in the context of the Quark system, which is similar to XPERANTO [23] in its support for querying XML views. We thus present an overview of XPERANTO, and also provide some background on XML and SQL triggers. We note that although our techniques are implemented in Quark, they are applicable to any XML publishing system.

\begin{tabular}{||l|l||}
\hline \hline Operator & Description \\
\hline Table & Represents a relational table \\
\hline Project & Computes results based on its input \\
\hline Select & Restricts its input \\
\hline Join & Joins two or more inputs \\
\hline Groupby & Applies aggregate functions and grouping \\
\hline Union & Unions inputs and removes duplicates \\
\hline Unnest & Applies super-scalar functions to input \\
\hline \hline & Table 1. XQGM operators. \\
\hline
\end{tabular}

\subsection{XPERANTO Overview}

In order to publish relational data as XML, XPERANTO first automatically creates a default view of the the relational data. The default view, which is not materialized, is a simple mapping from relational tables to XML elements. Users can create their own application-specific views by specifying the transformation from the default view using XQuery. As an example, consider a relational database and its default view shown in Figure 2 (the database contains products and vendors for each product; primary keys are capitalized). Now suppose this database is exposed as a (virtual) XML view in which vendors are nested under products, with the restriction that only products sold by at least two vendors appear in the view. The XQuery view definition corresponding to this view is shown in Figure 3, and it is materialized in Figure 4.

While there are many details about query processing in XPERANTO that are not relevant here, one important relevant aspect is XQGM (the XML Query Graph Model). $\mathrm{XQGM}$ is used to represent and manipulate XQuery queries and views. XQGM consists of a set of operators and functions. The set of operators is shown in Table 1. Each operator produces a set of output tuples whose column values are XML nodes/values. Various functions can be embedded in operators to represent the manipulation of XML nodes.

As an illustration, the XQGM graph for the view definition in Figure 3 is shown in Figure 5. Operators (boxes) 1 and 2 produce the tuples in the product and vendor tables, respectively. Box 3 joins each vendor with the product it sells, and box 4 constructs a $<$ vendor $>$ element for each of these tuples. Box 5 then groups the elements by product name: the aggXMLFrag() function groups all the vendor elements in a group into a sequence, while the count function counts the number of vendors per group. Box 6 selects only the tuples with count $\geq 2$. Finally, boxes $7-9$ create a $<$ product $>$ element for each product, group these into a single sequence, and produce a $<$ catalog $>$ element containing this sequence. 


\begin{tabular}{|c|c|c|c|c|}
\hline \multicolumn{4}{|c|}{ product } & \multirow{14}{*}{ 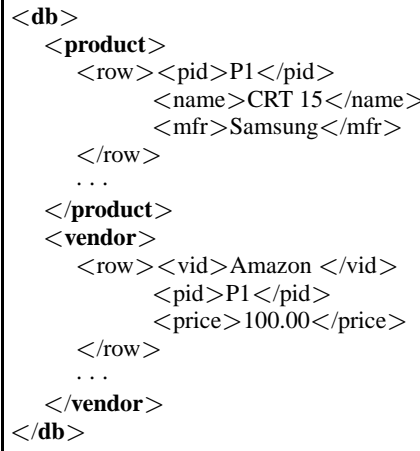 } \\
\hline PID & \multicolumn{2}{|c|}{ pname } & $\mathrm{mfr}$ & \\
\hline P1 & \multicolumn{2}{|c|}{ CRT 15} & Samsung & \\
\hline $\mathrm{P} 2$ & \multicolumn{2}{|c|}{ LCD 19} & Samsung & \\
\hline $\mathrm{P} 3$ & \multicolumn{2}{|c|}{ CRT 15} & Viewsonic & \\
\hline \multicolumn{4}{|c|}{ vendor } & \\
\hline \multicolumn{2}{|c|}{ VID } & PII & price & \\
\hline \multicolumn{2}{|c|}{ Amazon } & P1 & 100.00 & \\
\hline \multicolumn{2}{|c|}{ Bestbuy } & P1 & 120.00 & \\
\hline \multicolumn{2}{|c|}{ Circuitcity } & P1 & 150.00 & \\
\hline \multicolumn{2}{|c|}{ Buy.com } & P2 & 200.00 & \\
\hline \multicolumn{2}{|c|}{ Bestbuy } & $\mathrm{P} 2$ & 180.00 & \\
\hline \multicolumn{2}{|c|}{ Bestbuy } & P3 & $\overline{120.00}$ & \\
\hline \multicolumn{2}{|c|}{ Circuitcity } & P3 & 140.00 & \\
\hline
\end{tabular}

Figure 2. Example database and its default view.

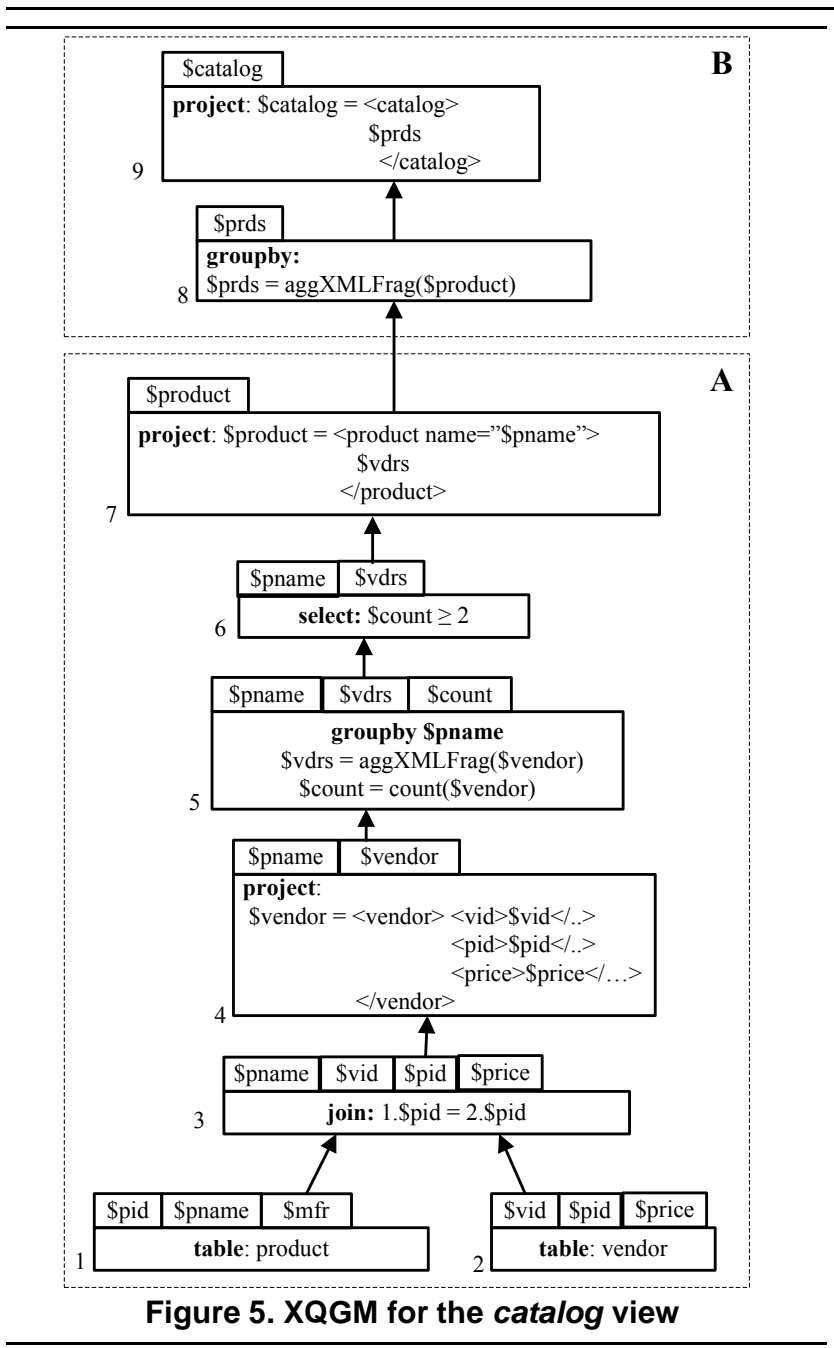

\subsection{XML Trigger Specification Language}

We use a subset of the trigger specification language proposed by Bonifati et al. [2], whose syntax is shown below:

CREATE TRIGGER Name AFTER Event

ON Path WHERE Condition DO Action

A trigger has a unique Name. The Event specifies the

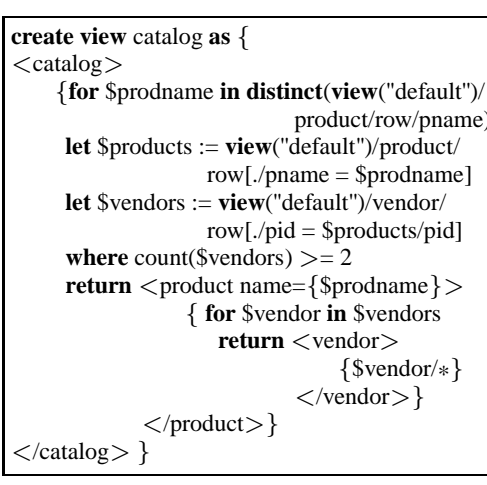

\{for \$prodname in distinct(view("default")/ product/row/pnam

let \$products := view("default")/product/ row $[. /$ pname $=\$$ prodname $]$ let \$vendors := view("default")/vendor/ row[./pid $=\$$ products $/$ pid $]$ where count $(\$$ vendors $)>=2$ return $<$ product name $=\{\$$ prodname $\}>$ $\{$ for $\$$ vendor in \$vendors return $<$ vendor $>$$$
\{\$ \text { vendor } / *\}
$$
$</$ catalog $>$ \}

$$
\underset{\}}{</ \text { product }>\}}</ \text { /vendor }>\}
$$



Figure 3. XML view definition.

operation that activates the trigger, and can be either UPDate, Insert, or Delete. Path is an XPath expression that specifies the portion of the XML view that is to be monitored for the event. Condition is a Boolean XQuery expression that specifies the condition under which the trigger is to be fired. When the condition is satisfied, the Action is performed; in our system, the action is a call to an external function which takes in XQuery expressions as parameters. Finally, two variables, OLD_NODE and NEW_NODE, are bound to the value of the node specified by Path before and after the Event; they may be referenced in the Condition and the Action. (When the Event is INSERT or DELETE, only the NEW_NODE or OLD_NODE, respectively, can be used.)

An example trigger over the view in Figure 3 is shown below. On any update to a product whose name was "CRT 15 " (before the update), the trigger invokes an external function notifysmith () with the new value of that product. Note that the trigger will be fired not only for direct updates to a <product > element, but also for updates to its descendant nodes (i.e. vendors selling that product).

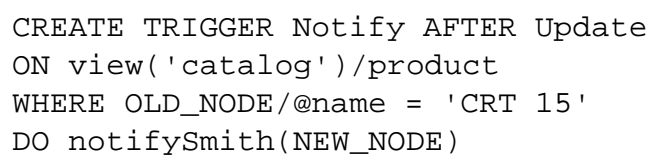

\subsection{SQL Triggers}

In contrast to XML triggers, which are specified on XML nodes, SQL triggers [6,7] are fired when an event (INSERT, UPDATE, or DELETE) occurs on a specific relational table. When an SQL trigger is activated, it has access to the before-update and after-update versions of the affected rows through transitional tables. We use the notation $\nabla$ table to denote the transitional table that contains the updated rows before an update, and $\triangle$ table to denote the transitional table that contains the updated rows after an update $(\nabla$ table is empty for INSERT triggers, and $\triangle$ table is empty for DELETE triggers). For example, if product P 1 goes on sale at Amazon, then the transitional tables might look like: 


\begin{tabular}{|c|c|c|}
\multicolumn{3}{|c}{$\nabla$ vendor } \\
\hline vid & pid & price \\
\hline Amazon & P1 & 100.00 \\
\hline
\end{tabular}

\begin{tabular}{|c|c|c|}
\multicolumn{4}{|c}{$\triangle$ vendor } \\
\hline vid & pid & price \\
\hline Amazon & P1 & 75.00 \\
\hline
\end{tabular}

\section{Semantics and System Architecture}

We now formalize the semantics of triggers on views, and then present our system architecture.

\subsection{Semantics of Triggers on XML Views}

In order to define the semantics of triggers on views, we need a precise definition of when an XML element in a view is said to be updated, inserted, or deleted. This in turn requires us to define the identity of an element in the view (so that we can talk about that element being updated, inserted or deleted). Note that the issue of identity is not as problematic for triggers over native XML data because each physical XML element has a well-defined notion of identity based on the XML data model. In contrast, XML elements in views are virtual and do not have a standard notion of identity. We now present an intuitive definition of the identity of XML elements based on the semantic structure of a view (in terms of the view's XQGM graph). The main idea is to use the notion of keys of XQGM operators to define the identity of nodes.

Definition 1 (Keys of XQGM Operators). Given an operator o in XQGM graph G, a key of o is a is a minimal set of (existing or derivable) columns of o whose values uniquely identify each output tuple produced by o.

As an illustration, a key of the table operator in box 1 in Figure 3 is the pid column (which is the product table's relational primary key). A key of the project operator in box 7 is the column containing the \$pname values (since the operator produces an output for each unique \$pname). Note that this key column is not directly present in the project operator but can be derived from its input operator.

In general, an operator can have more than one key. For instance, a relational table can have one column be a primary key and have a unique constraint on a different column. In such cases, we pick one of the keys to be the canonical key. For the table operator, we choose the primary key. The canonical keys for the other XQGM operators can be defined in terms of the canonical keys of its input operators (See Appendix A). For a tuple $t$ produced by an operator $o$, we use the notation $v(t)$ to denote the value of (all columns of) $t$. We write $c k v_{o}(t)$ to denote the value of the canonical key columns of $o$ for the tuple $t$. We denote the top operator of an XQGM graph $G$, which produces the final result of the graph, as $o_{G}$.

In order to define updates, inserts, and deletes on a view, we first formalize the notation for a database transition, which is the result of UPDATES, INSERTs, and/or DELETES on relational tables. We do so in terms of the database state, where the database is in a state $D$ before the transition, and a different state $D^{\prime}$ after the transition; we write the transition itself as $D \stackrel{*}{\rightarrow} D^{\prime}$. When considering the effect of UPDATES, INSERTs, and/or DELETEs to a single table $T$ (as is the case when a SQL trigger on $T$ is fired), we denote the transition as $D \stackrel{T}{\rightarrow} D^{\prime}$. The result of evaluating operator $o$ in state $D$ is written $R(o, D)$.

We now define updates, inserts and deletes on views.

Definition 2 (View Trigger Updates). A tuple $t$ is said to be updated in view $G$ by relational transition $D \stackrel{*}{\rightarrow} D^{\prime}$ iff $t \in R\left(o_{G}, D\right)$, and $\exists t^{\prime}\left(t^{\prime} \in R\left(o_{G}, D^{\prime}\right) \wedge c k v_{o_{G}}(t)=\right.$ $\left.c k v_{o_{G}}\left(t^{\prime}\right) \wedge v(t) \neq v\left(t^{\prime}\right)\right)$.

Definition 3 (View Trigger Inserts (Deletes)). A tuple $t$ is said to be inserted (deleted) in view $G$ by relational transition $D \stackrel{*}{\rightarrow} D^{\prime}\left(D^{\prime} \stackrel{*}{\rightarrow} D\right)$ iff $t \in R\left(o_{G}, D^{\prime}\right)$, and $\neg \exists t^{\prime}\left(t^{\prime} \in R\left(o_{G}, D\right) \wedge c k v_{o_{G}}(t)=c k v_{o_{G}}\left(t^{\prime}\right)\right)$.

Given the above definition of events, we use the semantics of XML triggers specified by Bonifati et al. [2]. Note that our events are well-defined only for operators with (canonical) keys. We thus need to define a class of views for which triggers are well-defined.

Definition 4 (Trigger-Specifiable Views). A view with $X Q G M$ graph $G$ is trigger-specifiable iff every operator in $G$ has a (canonical) key.

We require every operator (not just the top operator) in the view to have a canonical key because the user can specify a trigger on a nested element (and not just a top level element). We can prove the following theorem (See Appendix B).

Theorem 1: A view $G$ is trigger-specifiable if all the table operators in $G$ have (canonical) keys.

Thus, arbitrarily complex views can have triggers specified on them, so long as the underlying relational tables have primary keys (which is the common case). We can also relax this restriction for a certain class of views and triggers but we do not describe these relaxations here.

\subsection{System Architecture}

Our system architecture is shown in Figure 6. Users can create triggers (using the syntax in 2.2) on triggerspecifiable views. The Path, Condition and Action of the trigger are converted into their respective XQGM graphs (recall that Path, Condition and Action are all XPath or XQuery expressions, and hence can be converted to XQGM). The trigger Event and the Path graph are then analyzed by the Event Pushdown module to determine the minimal set of base relations on which inserts, updates, or deletes could cause the trigger to be fired. 


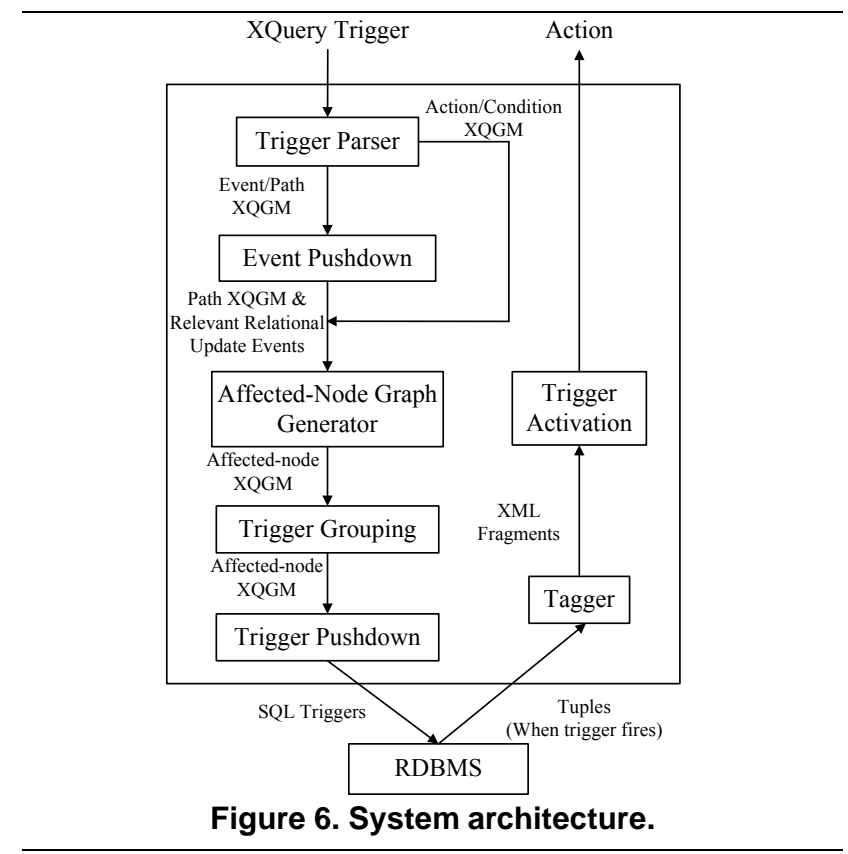

For each of these tables, the Affected-Node Graph Generator constructs an XQGM graph which, when evaluated, produces the OLD_NODE and NEW_NODE values for each affected XML node. This graph is then fed into the Trigger Grouping module, which groups similar triggers together for improved scalability. The Trigger Pushdown module takes the grouped trigger graph, pushes down selection conditions, and produces a set of SQL triggers, one for each relational event.

When activated, an SQL trigger issues a single SQL query to retrieve the relational data required for the actions of the XML triggers. The constant-space Tagger [23] then converts these results to XML. Finally, the Trigger Activation module activates the appropriate XML triggers and passes in the XML results as parameters to their actions.

In our implementation, we support a powerful subset of XQuery. Specifically, we support arbitrarily complex nested views with FLWOR expressions, quantified expressions, XPath expressions with child/descendant axes, arithmetic operators, comparison operators, and element constructors. We do not support XQuery type expressions or sibling / parent / ancestor XPath axes. For XML triggers, the Path, Condition and parameters to the Action can also be arbitrarily complex XQuery expressions with the same restrictions as for XML views. The grammar of supported expressions is specified in Appendix D. We note that our restrictions on XQuery expressions are an artifact of our current implementation and not an inherent limitation of our architecture. Also, while our system is implemented as middleware on top of a relational database, it can also be integrated into a database with XML publishing support.

Finally, a limitation of our current implementation is that
XML trigger(s) are fired for each SQL INSERT, UPDATE, or DELETE statement, rather than for each SQL transaction (which could contain more than one statement). This is not a limitation of our approach itself, but due to the fact that most commercial databases do not support SQL triggers at the transaction level; they only support SQL triggers at the granularity of a statement within a transaction. We note that our approach is general enough to support transaction level $\mathrm{XML}$ triggers if the underlying relational databases exposes transaction level SQL triggers.

\subsection{Trigger Parsing and Event Pushdown}

The first step in our architecture is to convert the trigger Path, Condition and Action XQuery expressions into XQGM; this is done in a manner similar to converting XQuery views to XQGM (see Section 2.1). In addition, we apply view composition rules [23] on the Path expression to identify the specific part of the view that the trigger monitors. For example, the trigger in Section 2.2 monitors the path view( 'catalog')/product. On composing this path with the catalog view, it produces the XQGM graph in Figure 5A. Note that this graph only produces products and not the entire catalog (since the trigger only monitors products).

The next step is to determine which events on which relational tables can cause the event specified in the XML trigger. This is similar to the problem of identifying events on the base tables that can affect materialized views [4] and violate constraints [3]. We adopt a similar approach to identifying relevant events on base tables and present the details in Appendix C. In our example, we are interested in UPDATE on the result of Box 7 in Figure 5A; this can be caused either by an UPDATE on the product table, or by an INSERT, UPDATE or DELETE on the vendor table.

\section{Affected-Node Graph Generation}

The goal of the Affected-Node Graph Generation module (see Figure 6) is to produce XQGM graphs that compute the input parameters for the trigger action. Specifically, the module takes as input the XQGM graphs for the Path, Condition, and parameters for the Action, and also the set of relational tables identified by the Event Pushdown module. For each of these tables, it produces an XQGM graph that computes the transformation from the relational transition tables to the parameters for the trigger action.

Our high-level approach is to produce a single XQGM graph, $G_{\text {params }}$, consisting of three parts, as shown in Figure 7. $G_{\text {affected }}$ produces a (OLD_NODE, NEW_NODE) tuple for each affected node of the view. $G_{c o n d}$, the XQGM graph corresponding to the Condition, evaluates the condition predicate which is then used to filter out any tuples that do not satisfy the condition. $G_{\text {action }}$ then computes the 


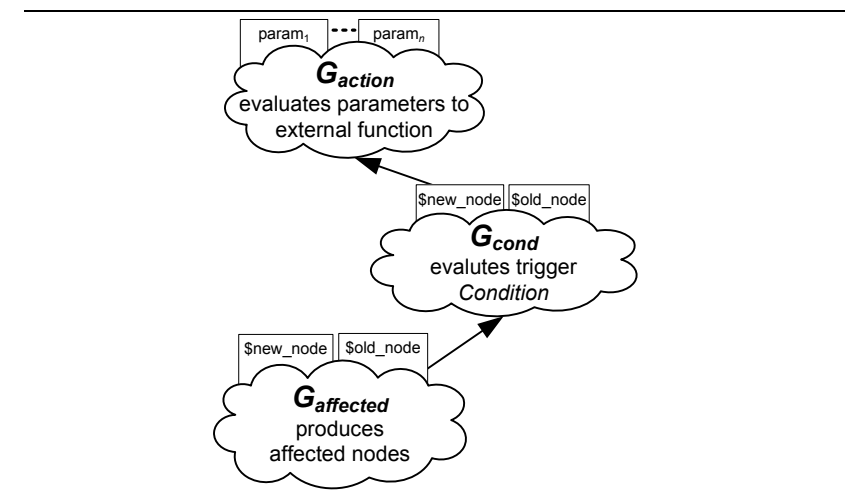

Figure 7. $G_{\text {params }}$ : Producing parameters to Action.

XQuery expressions given as parameters to the Action. The main technical contribution of this section is an algorithm to produce $G_{\text {affected }}$.

\subsection{Technical Challenges in Producing $G_{\text {affected }}$}

On the surface, the problem of producing $G_{\text {affected }}$ may appear similar to the incremental view maintenance problem (where the goal is to compute changes to a materialized view based on updates to the base data). However, there are three new challenges that arise in our context, which require the development of new techniques.

First, as mentioned in the introduction, one of our design goals is to not materialize the XML view. We avoid materialization because (a) it would require an additional sophisticated XML database that can support incremental view updates, and (b) it would require the view to be updated for every relevant relational update even though user triggers may have very selective predicates ${ }^{1}$. In contrast, most incremental view maintenance algorithms (e.g., [1, 4, 9, 10, $12,15,16,21])$ assume that the view is materialized, and use the materialized old value of a data item to compute its new value. We thus need to devise techniques that can directly compute the relevant new values from the base data.

Second, in producing $G_{\text {affected }}$, we need to compute new and old values after an update. In contrast, only the new value needs to be computed for materialized views. Thus, even materialized view techniques that can compute new values without using materialized old values (e.g., $[18,19])$ are not directly applicable because they cannot compute (old value, new value) pairs. This problem is especially acute for INSERT/DELETE events because they introduce specific restrictions on whether the old/new values can appear in the view before/after an update (Definition 3).

1 Note that if we chose to materialize the view, all items in the view (even those that do not satisfy any trigger selection predicate) would have to be incrementally maintained, because any item could become the old value of an updated item that does satisfy a trigger predicate.
Finally, the third (and perhaps most important) challenge arises due to nested predicates in XQuery. For instance, in Figure 5, we have multiple group-by (nesting) operators along with a selection predicate on a group-by aggregate value. While prior work on view maintenance for object-oriented [12, 16, 21], nested relational [15] and semistructured $[1,9,10]$ databases support nesting, they do not work with nested predicates. To understand why, consider the following example where a transaction inserts a row into the vendor table. The corresponding transition table is:

\begin{tabular}{|c|c|c|}
\multicolumn{3}{|c}{$\triangle$ vendor } \\
\hline vid & pid & price \\
\hline Amazon & P2 & 500.00 \\
\hline
\end{tabular}

Intuitively, for the XML view in Figure 5A, the above insert corresponds to an update of the "LCD 19" XML product (since a new vendor is added to this product). However, it turns out that the change computation technique (also referred to as the propagate phase [18]) commonly used for view maintenance will not detect this update. Specifically, most view maintenance algorithms compute changes to a view by replacing an updated table in the view definition with its corresponding transition table. In our example, this corresponds to replacing the vendor table in Figure 5A with the $\triangle$ vendor table, and evaluating the resulting query to compute the changes to the view. However, since $\triangle$ vendor has a single row, boxes 2,3 and 4 will each produce a single row and the selection in box 6 will return no rows since $\$$ count $=1$. Hence, no changes will be detected!

As the reader has probably observed, the above problem arises because we are trying to compute changes for nested predicate views using only tuples from the transition table. This results in inaccurate aggregate values and hence misses some relevant updates (it can also introduce spurious updates in other cases). We thus need to devise techniques for correctly computing changes for views with nested predicates. We note that [1] does present a technique for computing changes to views with existential predicates and a single level of nesting (existential predicates can be viewed as a very specific form of a select over an aggregation). However, we are not aware of any prior technique that can handle complex query predicates at arbitrary levels of nesting.

\subsection{Proposed Algorithm}

We now present our algorithm for producing $G_{\text {affected }}$. The algorithm first detects the keys of the XML nodes affected by an update (affected keys) and then use the affected keys to compute the actual node values. Our main contributions are (a) a technique for correctly determining affected keys even when the view has arbitrary nested predicates, and (b) a technique for using the affected keys to generate (OLD_NODE, NEW_NODE) pairs that satisfy the definition of trigger events, without using any materialized data. 
In what follows, we use the following notations. $G$ is the original Path graph; $B$ is the post-update version of the table in question (keep in mind that this algorithm is invoked once for each base relational table); $B_{\text {old }}$ is the pre-update version of this table; $G_{\text {old }}$ is a graph identical to $G$ with the sole exception that $B$ is replaced by $B_{\text {old }}$. While most DBMSes do not expose the $B_{\text {old }}$ table directly, it can easily be constructed using a query of the form [7]: (SELECT * FROM B) EXCEPT (SELECT * FROM $\triangle B$ ) UNION (SELECT * FROM $\nabla B$ ).

4.2.1. CreateAKGraph: Finding Affected Keys. Figure 8 presents our algorithm for determining the affected keys. The algorithm takes as input an operator $O$ (the top operator in the Path graph), a base table $T$, and a transitional table $d T$ (which is either $\triangle T$ or $\nabla T$ ). It returns a new operator, $O^{\prime}$, which is the top operator of an XQGM graph such that $O \bowtie O^{\prime}$ will produce exactly the subset of $O$ 's output tuples which are affected by the relational update captured by $d T$.

In order to determine the keys of $G$ affected by $\triangle B$ (or $\nabla B$ ), we traverse $G$ (or $G_{\text {old }}$, respectively) in depth-first order, building up a parallel graph $G_{\triangle k e y}$ (or $G_{\nabla k e y}$, respectively). At each step, we maintain the following invariant: for each operator $o$ in $G$ and the corresponding operator $o \triangle$ in $G_{\triangle k e y}$, joining $o$ and $o \triangle$ on the key of $o$ will produce exactly those tuples from the result of $o$ that were affected by $\triangle B$. Thus, if $o$ is the top operator of $G$, then the corresponding $o \triangle$ operator provides a way to identify the nodes in the result of $G$ that are affected by relational update.

We now walk through the algorithm using the Path graph in Figure 5A, for the case of an UPDATE on vendor (the other cases are similar). At the leaf level, a Table $(\triangle$ vendor $)$ operator will first be created. Clearly the invariant holds at this point: joining Table $(\triangle v e n d o r)$ with Table(vendor) on the \$vid column (the key of Table(vendor)) would produce exactly the vendor tuples that changed. The result after this step is shown in Figure 9.

Box 3 (the Join operator) merely propagates the \$vid column without creating any new operators in $G_{\triangle k e y}$. The corresponding operator in $G_{\triangle k e y}$ remains Table $(\triangle$ vendor $)$, and the invariant still holds: joining box 3 with $\triangle$ vendor on $\$$ vid would produce exactly those product-vendor pairs affected by $\triangle$ vendor. For box 4, we can similarly just propagate the \$vid column without having to create any new operators in $G_{\triangle k e y}$.

We then arrive at box 5, a GroupBy operator. Since a GroupBy operator aggregates multiple input values, any update to any one the input values in a group can change the aggregate result for that group. We therefore need a way to create an operator $o_{\triangle}$ in $G_{\triangle k e y}$ that only produces the keys of those groups affected by the update. First, we join the operator below the current GroupBy (box 4) with its
1: CreateAKGraph $(O, T, d T)$ : (Operator, Key)

$\{O$ is an operator; $T$ and dT are table names.

$I \leftarrow$ input operators to $O$

if $O . t y p e=$ Table then

if O.tableName $=T$ then

$P K \leftarrow$ primary key of table $\mathbf{T}$

$\left(O^{\prime}, K\right) \leftarrow\left(\operatorname{Project}_{(P K)}(\operatorname{Table}(d T)), P K\right)$

else

$\left(O^{\prime}, K\right) \leftarrow(\varnothing, \varnothing)$

end if

else if O.type $=$ GroupBy then

$I^{\prime} \leftarrow$ CreateAKGraph $(I, T, d T)$

if $I^{\prime}=\varnothing$ then

$\left(O^{\prime}, K\right) \leftarrow(\varnothing, \varnothing)$

else

$J \leftarrow \operatorname{Join}_{\left(k e y\left(I^{\prime}\right)\right)}\left(I, I^{\prime}\right)$

$K \leftarrow$ grouping columns of $O$

$O^{\prime} \leftarrow$ new GroupBy on $J$ with grouping cols $K$ end if

else if $O$. type $=$ Select or $O . t y p e=$ Project then

$\left(I^{\prime}\right.$, key $\left._{I}\right) \leftarrow$ CreateAKGraph $(I, T, d T)$

$\left(O^{\prime}, K\right) \leftarrow\left(I^{\prime}\right.$, key $\left._{I}\right)$

else if $O$ type $=$ Join then

\{Assuming, without loss of generality, that $|I|=2$.

for all $i \in I$ do

$\left(i^{\prime}, k\right) \leftarrow$ CreateAKGraph $(i, T, d T)$

if $i^{\prime} \neq \varnothing$ then

$I^{\prime} \leftarrow I^{\prime} \cup\left\{i^{\prime}\right\}$

$K \leftarrow K \cup\{k\}$

end if

end for

if $\left|I^{\prime}\right|=0$ then

$O^{\prime} \leftarrow \varnothing$

else if $\left|I^{\prime}\right|=1$ then

$O^{\prime} \leftarrow I^{\prime}$

else

\{Create a union of cross-products

$J_{a} \leftarrow \operatorname{Project}_{(K)}\left(\operatorname{Join}\left(I_{0}^{\prime}, I_{1}\right)\right)$

$J_{b} \leftarrow \operatorname{Project}_{(K)}\left(\operatorname{Join}\left(I_{0}, I_{1}^{\prime}\right)\right)$

$O^{\prime} \leftarrow \operatorname{Union}\left(J_{a}, J_{b}\right)$

end if

else if O.type $=$ Union then

\{Assuming, without loss of generality, that $|I|=2$.

for all $i \in I$ do

$\left(i^{\prime}, k\right) \leftarrow$ CreateAKGraph $(i, T, d T)$

if $i^{\prime} \neq \varnothing$ then

$I^{\prime} \leftarrow I^{\prime} \cup i^{\prime}$

$K_{i} \leftarrow k$

end if

end for

end for
\{Construct the key, as described in Appendix A\}

$K \leftarrow \bigcup_{i \in I} \bigcup_{c \in K_{i}} M(c)$

$\left\{\right.$ Create union of all operators in the set $\left.I^{\prime}\right\}$

$O^{\prime} \leftarrow \operatorname{Union}\left(I^{\prime}\right)$

end if

\{Ensure that $O$ 's key is propagated

Add $K$ to O.outputColumns

Return $\left(O^{\prime}, K\right)$

Figure 8. Algorithm for producing affected keys.

corresponding operator in $G_{\triangle k e y}\left(1_{\triangle}\right)$. By the algorithm invariant, we can infer that this new join produces exactly the set of input values to the GroupBy operator that have changed. Thus, to identify the keys of all affected groups, we simply need to project distinct values of the grouping columns, which we achieve by creating a new GroupBy operator $(3 \triangle)$ which groups on this column (\$pname). The $G_{\triangle k e y}$ graph at this point is shown in Figure 10.

The final two operators are Select and Project operators and, like boxes 3 and 4, merely propagate the key col- 


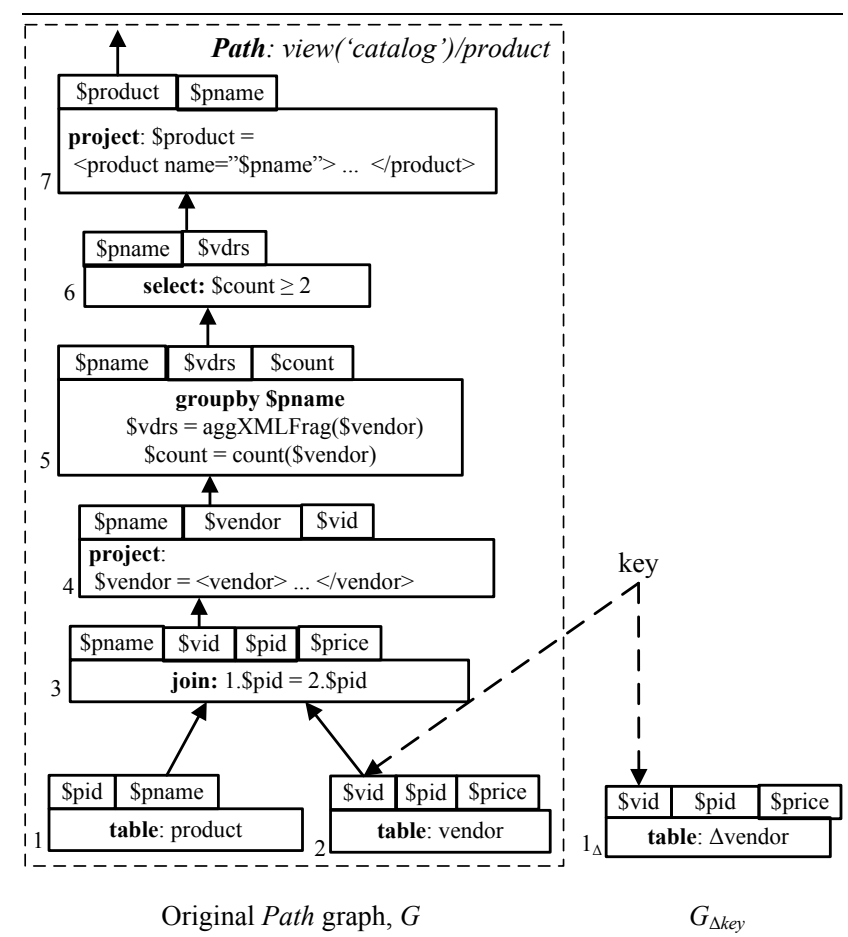

Figure 9. CreateAKGraph: Step 1.

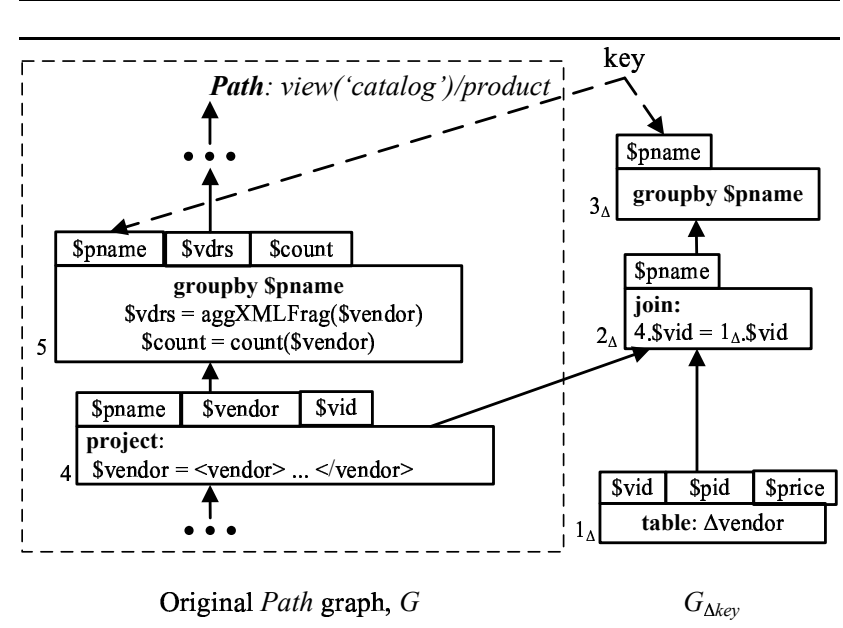

Figure 10. CreateAKGraph: GroupBy operator.

umn (\$pname); no new boxes are created in $G_{\triangle k e y}$. The final graph is shown in Figure 11. Note that the only difference from Figure 10 is that box 7 now propagates $\$$ pname.

The application of GetAKGraph is virtually identical for producing $G_{\nabla k e y}$, so we don't walk through it here; the only difference is that vendor ${ }_{\text {old }}$ and $\nabla v e n d o r$ are used instead of vendor and $\triangle$ vendor, respectively.

\subsubsection{CreateANGraph: Producing Affected Nodes.} At this stage, for a given relational table-event pair, we have the Path graphs $\left(G\right.$ and $\left.G_{\text {old }}\right)$ and the affected-key graphs $\left(G_{\triangle k e y}\right.$ and $\left.G_{\nabla k e y}\right)$. Our goal now is to produce the $G_{\text {affected }}$ graph, which produces the (OLD_NODE,

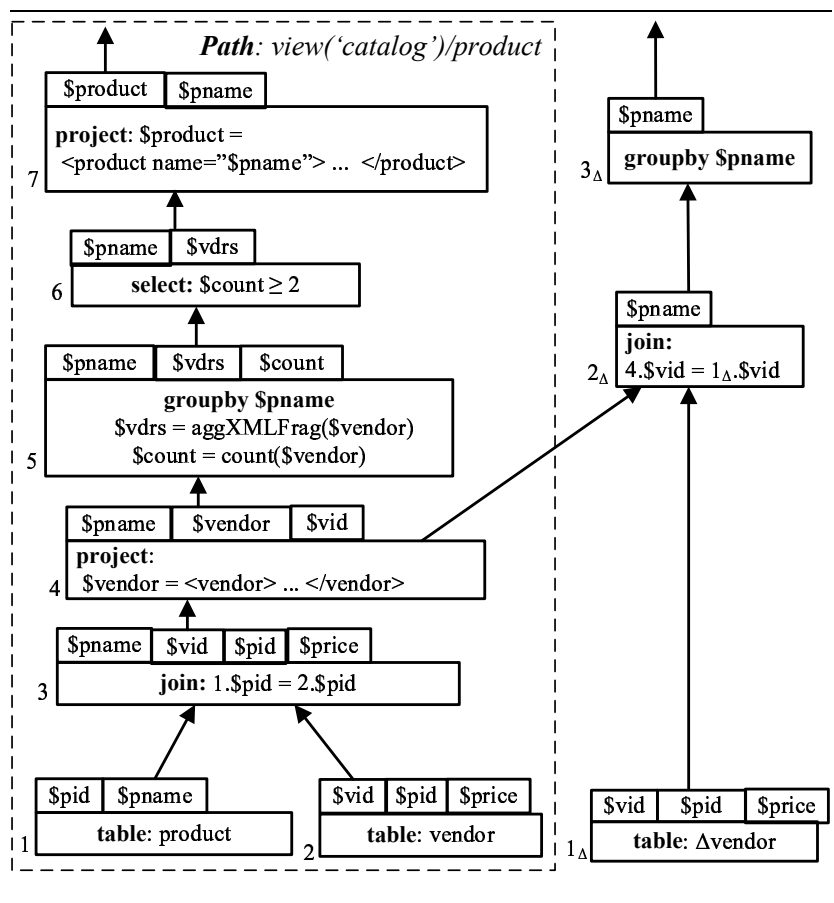

Original Path graph, $G$

$G_{\Delta k e y}$

Figure 11. The complete $G_{\triangle k e y}$ graph.

NEW_NODE) pairs corresponding to the relational update. The algorithm for producing $G_{\text {affected }}$ is given in Figure 12. We begin by creating a Union operator merging the $G_{\triangle k e y}$ and $G_{\nabla k e y}$ subgraphs. This captures the notion that we need to compute the (OLD_NODE, NEW_NODE) pairs for nodes affected by either insertions or deletions at the relational level. Next, the result of the union is joined with $G$ to get NEW_NODE, and joined with $G_{\text {old }}$ to get OLD_NODE. Finally, OLD_NODE and NEW_NODE are joined on the key. The type of this join depends on the XML trigger Event: an UPDATE has both OLD_NODE and NEW_NODE (hence an inner join), an INSERT has only NEW_NODE (hence a left anti join), and DELETE, only OLD_NODE (hence a right anti join). In our example, we perform an inner join since we are monitoring UPDATES. In some special cases of UPDATEs (not relevant to our example), we need to explicitly check whether the values of OLD_NODE and NEW_NODE are different; we refer the reader to Appendix $\mathrm{F}$ for more details of these special cases and related optimizations. Figure 13 shows the final $G_{\text {affected }}$ graph for our example.

We can prove the following correctness theorem for UPDATEs (and similarly for INSERTs and DELETES) (See Appendix E).

Theorem 2: Given an UPDATE event, view graph $G$, and table $T$, CreateANGraph produces graph $G_{\text {affected }}$ such that for all valid database transitions $D \stackrel{T}{\rightarrow} D^{\prime}$, 


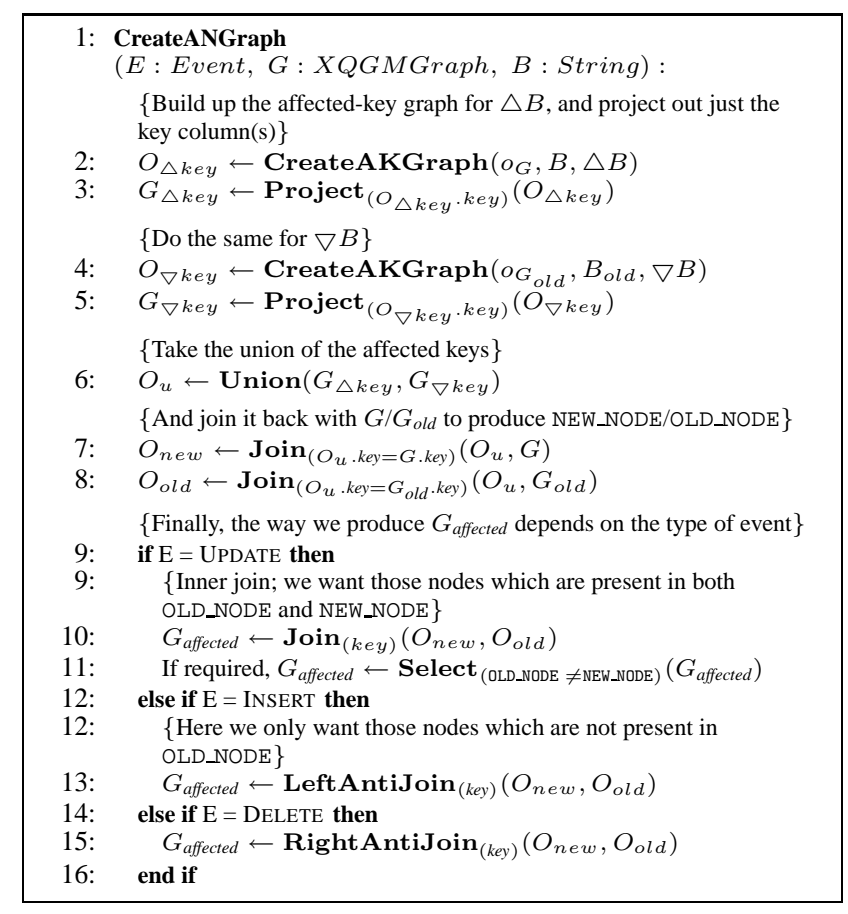

Figure 12. Algorithm for producing $G_{\text {affected }}$.

(OLD_NODE, NEW_NODE) $\in R\left(o_{G_{\text {affected }}}, D^{\prime}\right)$ iff OLD_NODE $\in$ $R\left(o_{G}, D\right) \wedge$ NEW_NODE $\in R\left(o_{G}, D^{\prime}\right) \wedge c k v_{o_{G}}($ OLD_NODE $)=$ $c k v_{O_{G}}($ NEW_NODE $) \wedge v($ OLD_NODE $) \neq v($ NEW_NODE $)$.

\subsection{Adding Condition and Action}

Finally, as described in the beginning of this section, we need to produce $G_{\text {params }}$, the graph that produces parameters for the Action after selecting only the (OLD_NODE, NEW_NODE) pairs that satisfy the trigger condition (see Figure 7). $G_{\text {params }}$ is produced by converting the XQuery expressions for Condition and parameters of Action into XQGM (to produce $G_{c o n d}$ and $G_{a c t i o n}$, respectively), and stacking these graphs on top of $G_{\text {affected }}$. Figure 13 shows $G_{\text {params }}$ for our running example.

\section{Trigger Grouping and Pushdown}

Given $G_{\text {params }}$ for each relevant table-event pair, the final two steps in generating SQL triggers are Trigger Grouping and Trigger Pushdown (Figure 6). We describe each in turn.

\subsection{Trigger Grouping}

A simple approach to producing SQL triggers is to create one SQL trigger for each $G_{\text {params }}$ graph of an XML trigger. However, this approach will not be very efficient because the number of SQL triggers produced will be at least as many as the number of XML triggers (which we expect

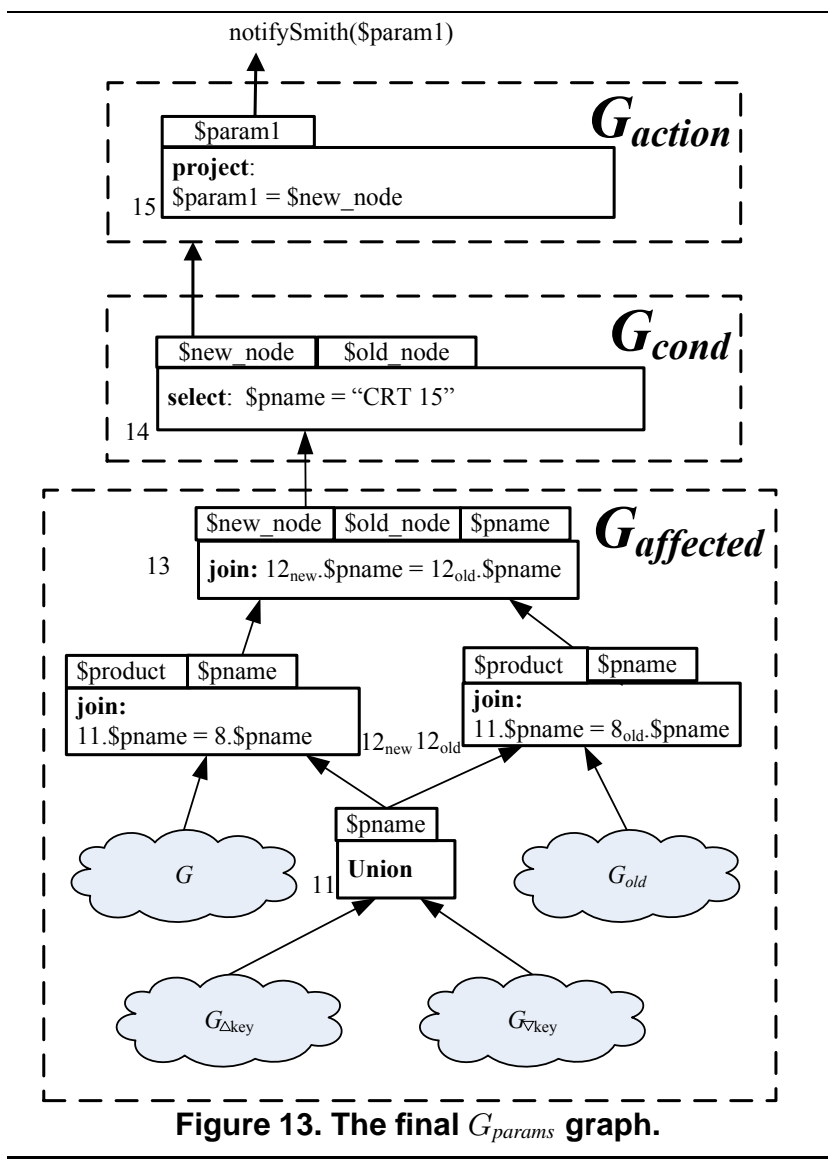

to be large), and current relational database systems are not very scalable with respect to the number of SQL triggers. We thus explore techniques for grouping structurally similar $G_{\text {params }}$ graphs together, and producing a single SQL trigger for each group. We note that our focus is not on developing new techniques for grouping triggers; rather, our focus in on adapting existing techniques $[5,14]$ to work with nested XML views.

For the purposes of this paper, we only consider grouping $G_{\text {params }}$ graphs that differ in the constant value(s) of a selection condition (this corresponds to grouping structurally similar XML triggers that only differ in selection constant(s) in the WHERE clause). For instance, we would consider grouping the $G_{\text {params }}$ graph in Figure 13 with another graph that is identical in all respects but for the constant value of the selection condition in box 14 (which could, say, select "LCD 19" instead). The proposed approach can also be extended for grouping joins, but we do not discuss this extension here.

The first step is to create a constants table [14] for each group of structurally similar $G_{\text {params }}$ graphs. The constants table has a TrigIDs column, which identifies the XML triggers which share a particular set of constants, followed by as many columns as there are constants in the triggers. For instance, if in our example, XML triggers 1 and 2 both share 
the value CRT 15, while trigger 3 uses LCD 19, the constants table would look like:

\begin{tabular}{|c|c|}
\hline TrigIDs & Const 1 \\
\hline 1,2 & CRT 15 \\
3 & LCD 19 \\
\hline
\end{tabular}

Given the constants table, the standard grouping technique $[5,14]$ is to directly convert the selection condition with constant(s) into a join with the constants table, as shown in Figure 14 for our example. In this way, multiple individual selections are converted into a single join, and are hence more efficient.

However, this direct replacement of a select with a join does not work for complex nested XML conditions. To see why, let us assume that the WHERE condition in our example is modified to be of the form: count(NEW_NODE/vendor[./price $<x]$ ) $\geq y$ (i.e., the new node contains at least $y$ vendors who sell an item for less than $x$; here $x$ and $y$ can be different constants for different XML triggers). In this case, the condition contains a selection $(. /$ price $<x)$ nested under a group-by (count) nested under another selection $(\geq y)$. Simply replacing a selection (such as ./price $<x$ ) with a corresponding join would be incorrect because this would change the output cardinality of the operator (due to the join with the constants table, where multiple triggers could be fired); this would in turn change the group-by (count) result, thereby producing wrong results.

To address this issue, we propose a simple yet powerful approach that works for arbitrarily complex nested selections. The basic idea is to use the constants table to set up a correlation in the $G_{\text {params }}$ graph to produce a $G_{\text {grouped }}$ graph, as shown in Figure 15. Conceptually, this means that the $G_{\text {params }}$ graph is evaluated once for each row in the constants table (i.e., for each unique set of constants). While this will certainly produce the correct results, it is likely to be inefficient because we still do selections one by one for each unique set of constants. However, the key idea now is to decorrelate this graph using query rewrite techniques developed for SQL [22] and XML [23] queries. Decorrelation converts correlated selections to joins $[22,23]$ (as we desire) and also preserves the correct semantics of the graph by adding appropriate grouping columns to group-by operators so that nested selections are handled correctly.

\subsection{Trigger Pushdown}

Given a decorrelated $G_{\text {grouped }}$ graph for a table-event pair, the next step is to generate a SQL trigger that, when activated, produces the output of $G_{\text {grouped }}$. In generating this SQL trigger, we leverage techniques developed for publishing relational data as XML. Specifically, we apply selection/join pushdown and tagger pull-up transformations [23] on $G_{\text {grouped }}$ to generate a single sorted outer union SQL

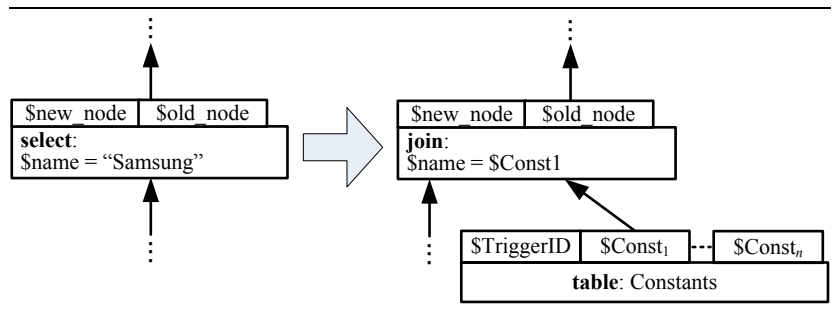

Figure 14. Converting select to join.

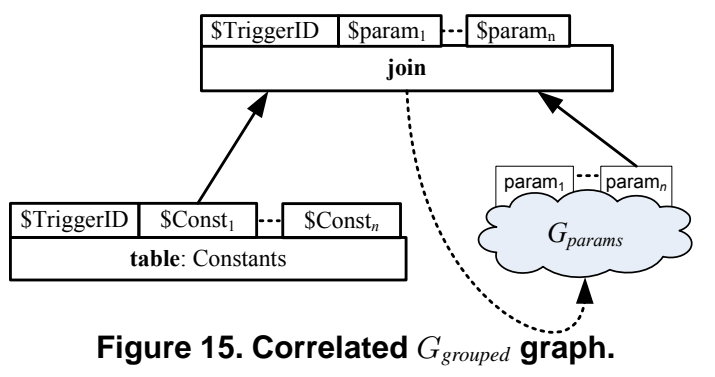

query whose results can be tagged in constant space to produce the XML output; this query becomes the body of the SQL trigger generated for the table-event pair.

We also employ an important optimization that is specific to triggers, which we now briefly describe. The main idea is to avoid directly computing distributive aggregate functions over $B_{\text {old }}$, the pre-update version of a table, whenever such functions appear in the view definition (such as a count over vendors, in our example). We perform this optimization for two reasons. First, when an aggregate function is specified in a view, we need to compute the aggregate for both $B$ (the post-update version of the table) and $B_{\text {old }}$ (the pre-update version of the same) since we need to produce both OLD_NODE and NEW_NODE values; computing an aggregate twice is likely to be expensive. Second, if we can avoid referencing $B_{\text {old }}$ directly, we might be able to avoid materializing $B_{\text {old }}$ (recall from Section 4.2 that $B_{\text {old }}$ is not exposed directly and has to be explicitly computed).

We use the following technique to avoid directly computing an aggregate on $B_{\text {old }}$, while still producing the correct OLD_NODE values. We compute the result of the aggregation on $B_{\text {old }}$ by using the result of the aggregation on $B$ and the transition tables. Note that this approach is exactly the inverse of the incremental view maintenance problem (since we compute old aggregate values from new aggregate values)! Consequently, by switching the role of old values and new values, we can directly use existing incremental view maintenance techniques [19] to compute aggregates on $B_{\text {old }}$ using just $B$ and the transition tables.

The SQL trigger generated for our running example of an UPDATE on vendor is shown in Figure 16 (formatted to be more human-readable). The trigger first finds the affected keys by taking a union of the product names associated with tuples in the transition tables (lines 6-13). The trigger then 


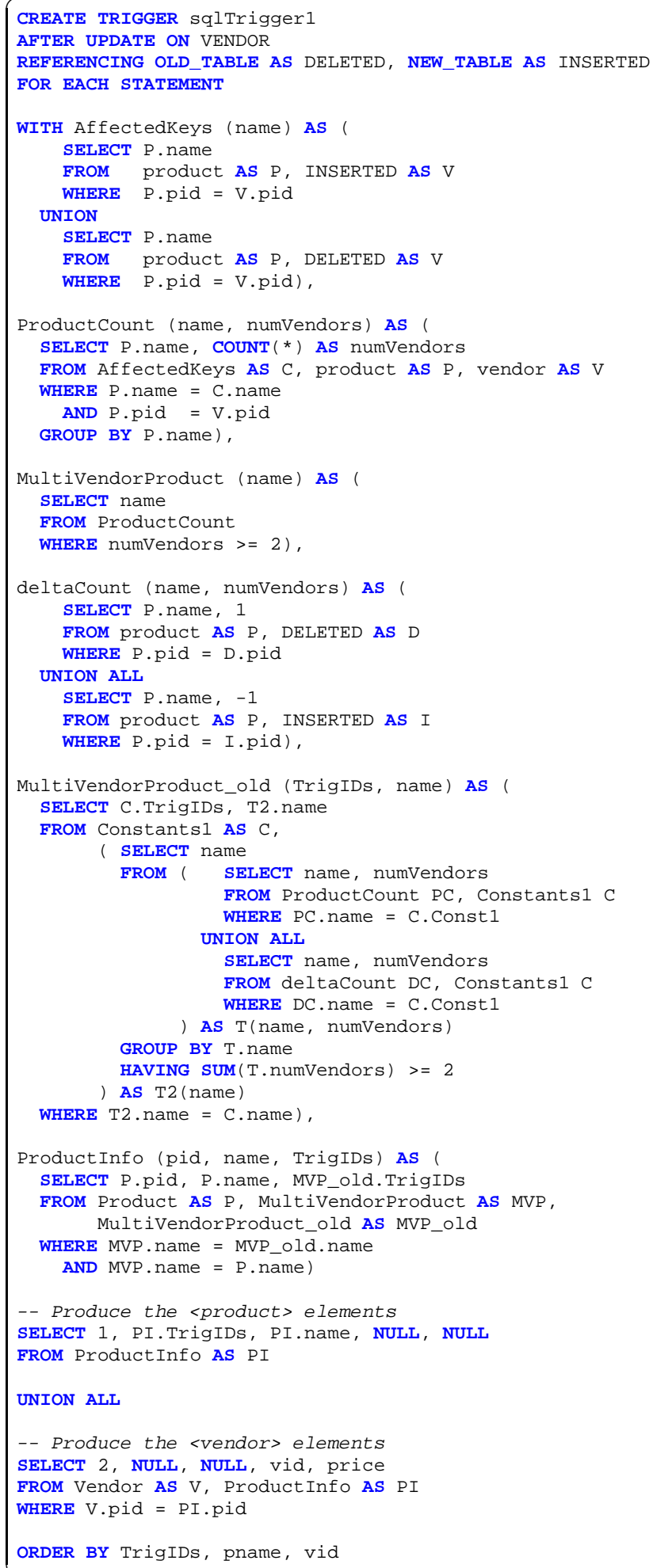

Figure 16. The generated SQL trigger.

\begin{tabular}{|l|l|}
\hline \hline Parameter & Values (default in bold) \\
\hline Hierarchy depth & $\mathbf{2 , 3 , 4 , 5}$ \\
\hline Number of leaf tuples $(\times 1000)$ & $32,64, \mathbf{1 2 8}, 256,512,1024$ \\
\hline Leaf tuples per XML element & $16,32, \mathbf{6 4}, 128,256$ \\
\hline Number of triggers & $1, \ldots, \mathbf{1 0 , 0 0 0}, \ldots, 100,000$ \\
\hline Number of satisfied triggers & $\mathbf{1 , 2 0 , 4 0 , 8 0 , 1 0 0}$ \\
\hline
\end{tabular}

Table 2. Experimental parameters.

computes the number of vendors for each affected product after the update (lines 15-20), and selects only those with more than one vendor as potential NEW_NODEs (lines 22$25)$. Note that vendors are only computed for affected products by using regular query rewrite techniques to push down the join on affected keys $[18,23]$. The trigger then computes the number of vendors for each affected product before the update by using the corresponding values after the update and the transition tables (lines 27-51); also note that the selection on the OLD_NODE name is transformed into a join due to trigger grouping. Finally, the action parameters are produced using a sorted outer union (lines 61-71).

\section{Experimental Evaluation}

We have developed and evaluated a prototype of the system architecture proposed in this paper. We observe that the compile time for an XML trigger, which is the time to manipulate the intermediate XQGM graphs and produce the final SQL trigger, is fairly small (a hundred milliseconds, even for a complex view), and is only expended once during the creation of the trigger. We therefore focus our evaluation on the run time, which is the overhead of evaluating the generated SQL trigger on an update to the underlying base table(s).

\subsection{Experimental Setup}

Our experimental setup is characterized by the parameters in Table 2. Hierarchy depth specifies the depth of the relational schema. For depth 2, we use the product/vendor schema and XML view described earlier. For deeper views, we add additional "ancestor" tables above product, so that each child table has a foreign key column referencing its parent's primary key, and the XML view contains children nested inside of parents. Number of leaf tuples specifies the number of rows in the leaf (i.e., vendor) table-this is a measure of the size of the database. Leaf tuples per XML element is the number of leaf tuples contained in a top-level XML element produced by the view; this is a measure of the size of an individual XML element. The next two parameters specify the number of structurally similar XML triggers in the system, and the number of these triggers that are fired for each relational update, respectively. In all cases, the XML trigger was placed on the top-level XML element 


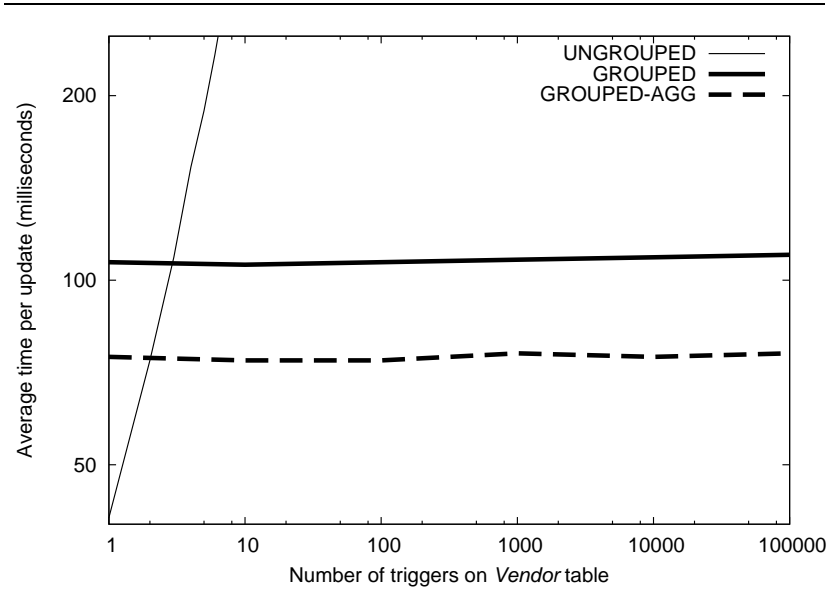

Figure 17. Varying the number of triggers.

in the view, and the $\operatorname{count}(\cdots) \geq 2$ predicate remained on the lowest level, that is, on the vendors. We defined the actions of the triggers to insert the entire NEW_NODE into a temporary table.

We evaluated three alternative implementations to evaluate the various aspects of our approach. UNGROUPED generates a different SQL trigger for each table-event pair corresponding an XML trigger. GROUPED extends UNGROUPED by implementing trigger grouping (Section 5.1). GROUPED-AGG extends GROUPED by optimizing aggregate computation on $B_{\text {old }}$ (Section 5.2). Our experiments were performed on a Linux system with a 933 MHz Pentium III processor and 1GB of main memory, running the DB2 8.1 commercial relational DBMS. We defined primary keys for all the relational tables and built appropriate indices on the key columns and other join columns. For each experiment, we varied one of the parameters in Table 2 and used default values for the rest (the default values are in bold). The run time was averaged over 100 independent updates to the vendor table, performed on a cold cache.

\subsection{Varying Number of Triggers}

Figure 17 shows the performance of the different approaches when we vary the number of triggers in the system. UNGROUPED does not scale well with the number of triggers because it does not benefit from shared computation across triggers. In contrast, GROUPED and GROUPED-AGG scale gracefully due to the grouping optimizations, with virtually no change in the update time as the number of XML triggers increases (note the log scale in the $\mathrm{X}$-axis). This suggests that we can build a scalable XML trigger system over relational databases, even though relational triggers by themselves are not scalable. Finally, GROUPED-AGG provides about a $30 \%$ performance improvement over GROUPED due to the ag-

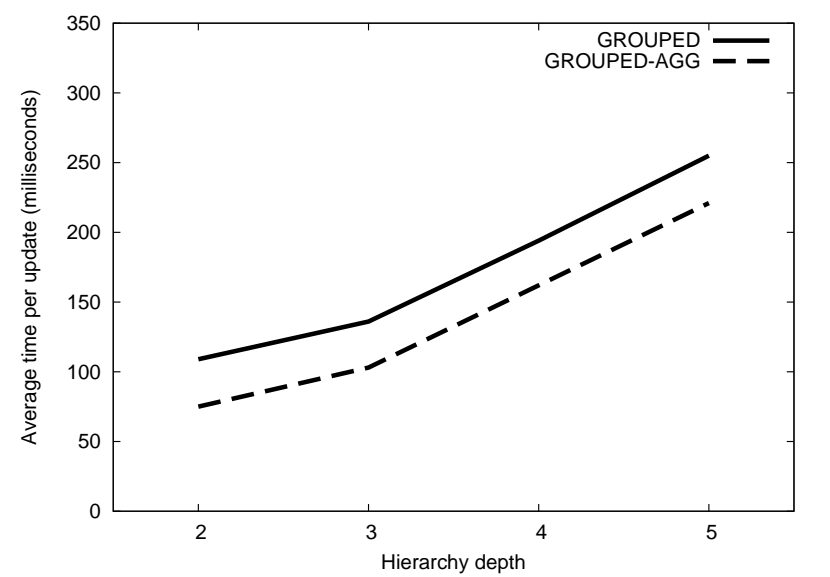

Figure 18. Varying the hierarchy depth.

gregation optimization.

\subsection{Varying Hierarchy Depth}

Figure 18 shows the effect of varying the depth of the XML view (we do not show UNGROUPED because of its bad scalability properties). GROUPED and GROUPED-AGG have the same relative performance and their run time increases approximately linearly with the depth. This is because, as the depth increases, the relational trigger must evaluate more joins to recreate the hierarchy. Further, the size of the produced result also increases because, although the number of leaf nodes remains constant, the number of intermediate nodes grows larger. The results indicate that we can get good performance for XML triggers even for deeply-nested XML views.

\subsection{Summary of Other Results}

We also varied the other parameters in Table 2 and we obtained similar results as in the previous two experiments: GROUPED and GROUPED-AGG scaled gracefully, and GROUPED-AGG provides at least a $30 \%$ improvement over GROUPED; this difference actually increases when we vary the number leaf tuples per XML element since the cost of aggregation increases. We refer the reader to Appendix G for further details.

\section{Related work}

There has been some recent work on supporting triggers in native XML databases [2, 17]. Our focus, however, is on implementing XML triggers using SQL triggers, without any need for a native XML database. A related class of systems is XML publish/subscribe (pub/sub) systems $[5,8,13,26,17]$, where the goal is to efficiently match 
streaming XML documents against a large set of subscriptions. However, most most pub/sub systems do not consider updates to XML documents, which is the main issue with XML triggers. Furthermore, even those that consider updates [17] work with native XML engines and do not exploit relational triggers. Our work is also related to the incremental maintenance of relational/nested-relational/objectoriented/semi-structured views; we refer the reader to Section 4.1 for the main technical differences with respect to view maintenance. In addition, our work also differs from XML view maintenance in that we exploit relational triggers and query processing.

\section{Conclusion and Future Work}

We have presented a systematic way of translating triggers over XML views of relational data into SQL triggers, and for translating relational updates into their corresponding XML updates. We have also presented experimental results that illustrate the feasibility and scalability of our approach. An interesting direction for future work is to see whether our general algorithm for detecting changes over complex XQuery views can be adapted for incrementally maintaining complex materialized XML views.

\section{References}

[1] S. Abiteboul, J. McHugh, M. Rys, V. Vassalos, J. Wiener, "Incremental Maintenance for Materialized Views over Semistructured Data", VLDB 1998.

[2] A. Bonifati, D. Braga, A. Campi, S. Ceri, "Active XQuery", ICDE 2002.

[3] S. Ceri, J. Widom, "Deriving Production Rules for Constraint Maintenance", VLDB 1990.

[4] S. Ceri, J. Widom, "Deriving Production Rules for Incremental View Maintenance", VLDB 1991.

[5] J. Chen, D. J. DeWitt, F. Tian, Y. Wang, "NiagaraCQ: A Scalable Continuous Query System for Internet Databases", SIGMOD 2000.

[6] R. Cochrane, K. Kulkarni, N. Mattos, "Active Database Features in SQL3", In N. Paton (ed.) Active Rules in Database Systems, Springer-Verlag, 1999.

[7] R. Cochrane, H. Pirahesh, N. Mattos, "Integrating Triggers and Declarative Constraints in SQL Database Systems", VLDB 1996.

[8] Y. Diao, P. Fischer, M. Franklin, R. To, "YFilter: Efficient and Scalable Filtering of XML Documents", ICDE 2002.

[9] K. Dimitrova, M. El-Sayed, E. Rundensteiner, "Order-sensitive View Maintenance of Materialized XQuery Views", ER 2003.

[10] M. El-Sayed, L. Wang, L. Ding, E. Rundensteiner, "An Algebraic Approach for Incremental Maintenance of Materialized XQuery Views", WIDM 2002.

[11] M. Fernandez, D. Suciu, W. Tan , "SilkRoute: Trading between Relations and XML”, WWW 2000.

[12] D. Gluche, T. Grust, C. Mainberger, M. Scholl, "Incremental Updates for Materialized OQL Views", DOOD 1997.

[13] A. Gupta, D. Suciu. "Stream Processing of XPath Queries with Predicates", SIGMOD 2003

[14] E. Hanson, C. Carnes, L. Huang, M. Konyala, L. Noronha, S. Parthasarathy, J. Park, A. Vernon, "Scalable Trigger Processing", ICDE 1999.

[15] A. Kawaguchi, D. Lieuwen, I. Mumick, K. Ross, "Implementing Incremental View Maintenance in Nested Data Models", DBPL 1997.
[16] H. Kuno, E. Rundensteiner, "Incremental Maintenance of Materialized Object-Oriented Views in MultiView: Strategies and Performance Evaluation", TKDE 10(5), 1996.

[17] B. Nguyen, S. Abiteboul, G. Cobena, M. Preda, "Monitoring XML Data on the Web", SIGMOD 2001

[18] T. Palpanas, R. Sidle, R. Cochrane, H. Pirahesh, "Incremental Maintenance for Non-Distributive Aggregate Functions", VLDB 2002.

[19] D. Quass, "Maintenance Expressions for Views with Aggregation", SIGMOD 1996.

[20] M. Rys, "State-of-the-Art Support in RDBMS: Microsoft SQL Server's XML Features", Data Engineering Bulletin 24(2), 2001.

[21] M. Rys, M. Norrie, H. Schek, "Intra-Transaction Parallelism in the Mapping of an Object-Model to a Relational Multi-Processor System", VLDB 1996.

[22] P. Seshadri, H. Pirahesh, T. Leung, "Complex Query Decorrelation", ICDE 1996.

[23] J. Shanmugasundaram, J. Kiernan, E. Shekita, C. Fan, J. Funderburk. "Querying XML views of relational data", VLDB 2001

[24] M. Stonebraker, A. Jhingran, J. Goh, S. Potamianos, "On Rules, Procedures, Caching and Views in Data Base Systems", SIGMOD 1990.

[25] D. Suciu, "Query Decomposition and View Maintenance for Query Languages for Unstructured Data”, VLDB 1996.

[26] F. Tian, B. Reinwald, H. Pirahesh, T. Mayr, J. Myllymaki, "Implementing A Scalable XML Publish/Subscribe System Using Relational Database Systems", SIGMOD 2004.

[27] World Wide Web Consortium. "XQuery 1.0: An XML Query Language", W3C Working Draft, 12 Nov. 2003. See http: / /www.w3.org/TR/2003/WD-xquery-20031112/.

[28] World Wide Web Consortium. "XQuery 1.0 and XPath 2.0 Functions and Operators", W3C Working Draft, 12 Nov. 2003. See http://www.w3.org/TR/2003/wD-xpath-functions$20031112 /$.

[29] X. Zhang, M. Mulchandani, S. Christ, B. Murphy, E. Rundensteiner, "Rainbow: mapping-driven XQuery processing system", SIGMOD 2002. 


\section{A. Definitions of Canonical Keys}

Table 3 defines the canonical key for each XQGM operator (except for Unnest) in terms of the canonical keys of its input operator(s).

\section{B. Proof of Theorem 1}

Theorem 1. A view of relational data, $G$, is triggerspecifiable if all the table operators in $G$ have (canonical) keys.

Proof. We need to prove that every operator in $G$ has a canonical key. In Table 3, we define the canonical keys for every type of operator, except for Unnest, in terms of its input operator(s). Thus, if $G$ does not contain any Unnest operators, then we can simply derive the canonical key for each operator $o$ by applying the definitions in Table 3 .

If $G$ does contain Unnest operators, then it can be rewritten to an equivalent graph $G^{\prime}$ that does not contain any Unnest operators. This transformation is possible because $G$ is an XML view of relational data and the underlying relational data contains no inherent nesting. Hence, an Unnest operator can only unnest an XML hierarchy created in the view itself. We can thus use the sound and complete view composition rules from [23] to remove Unnest operators in such cases. We can thus assume without loss of generality that $G$ does not contain any Unnest operators.

Since all operators in $G$ have canonical keys, the view is trigger-specifiable by Definition 4.

\section{Event pushdown}

In this part, we show our algorithm for determining what relational events can cause $X M L$ triggers to be fired.

First, we precisely define the notion of INSERT, UPDATE, and DELETE events on an operator $o$, given a database transition $D \stackrel{*}{\rightarrow} D^{\prime}$ :

- Insert $(o)$ : There exists $t \in R\left(o, D^{\prime}\right)$ such that $\neg \exists t^{\prime}\left(t^{\prime} \in R(o, D) \wedge c k v_{o}(t)=c k v_{o}\left(t^{\prime}\right)\right)$.

- $\operatorname{Delete}(o)$ : There exists $t \in R(o, D)$ such that $\neg \exists t^{\prime}\left(t^{\prime} \in R\left(o, D^{\prime}\right) \wedge c k v_{o}(t)=c k v_{o}\left(t^{\prime}\right)\right)$.

- Update $(o, C)$ : There exists $t \in R(o, D)$ such that for the set of columns $C, \exists t^{\prime}\left(t^{\prime} \in R\left(O, D^{\prime}\right) \wedge c k v_{o}(t)=\right.$ $\left.c k v_{o}\left(t^{\prime}\right) \wedge \pi_{C}(v(t)) \neq \pi_{C}\left(v\left(t^{\prime}\right)\right)\right)$.

The procedure we use is quite straightforward: for each type of operator (Join, GroupBy, etc.), and for each of the three event types, there is a set of possible input events which can directly cause that output event (see Table 4). Put another way, there is a set of rules $E_{I} \rightarrow E_{O}$, where operator $I$ is an input to operator $O$, such that the event $E_{O}$ can

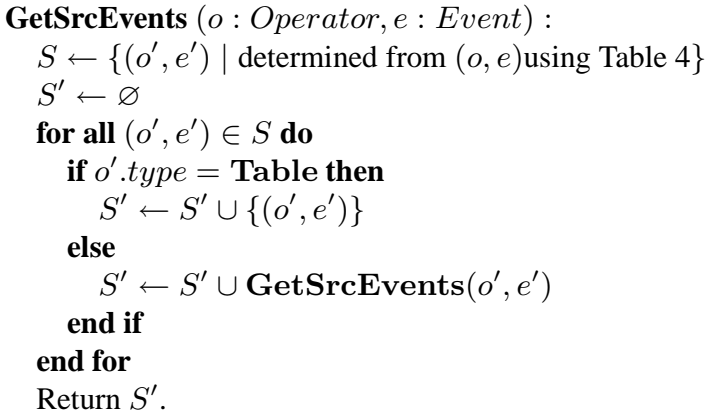

Figure 19. GetSrcEvents: given an operator, $o$, and a desired event on that operator, $e$, returns the set of table-level events which can cause $e$.

occur if $E_{I}$ occurs. Thus, starting at the top operator of the Path graph, we can determine the set of events on all of its input operators which can directly cause the Event specified in the trigger. Applying these rules recursively, as shown in Figure 19, we eventually reach the base Table operators, at which point we have the set of all base-table events $I_{B}$ such that $I_{B} \rightarrow$ Event.

\section{Supported XQuery Expressions}

Our implementation supports a powerful subset of XQuery in view and trigger definitions. As mentioned in Section 2.2, the trigger's Path is an XPath expression, while the Condition as well as the parameters to the $A c$ tion function are XQuery expressions. Rather than list the full XPath and XQuery grammar we support, we will instead highlight the differences between the grammar supported by our implementation and the XQuery $1.0 \mathrm{spec}-$ ification [27].

Figure 20 shows the restrictions we place on the XQuery 1.0 grammar. The two main restrictions are on the axes supported (we do not support parent or sibling accessors), and on type expressions, which we do not support (hence the removal of InstanceofExpr). There are also restrictions on the XQuery functions [28] which we support; currently, we allow only arithmetic functions which have counterparts in SQL, and we do not allow user-defined XQuery functions.

\section{E. Proof of Theorem 2}

In this section, we will prove the correctness of the CreateANGraph algorithm (see Figure 12). 


\begin{tabular}{|c|c|c|}
\hline Operator type & Input (operator, key) pairs & How to derive output key $\left(\mathrm{Key}_{O}\right)$ \\
\hline Select, Project & $\left(I\right.$, Key $\left._{I}\right)$ & $\begin{array}{l}\text { l* Simply propagate the key of our input operator. */ } \\
\mathrm{Key}_{O} \leftarrow K e y_{I}\end{array}$ \\
\hline Join & $\left(I_{1}, \operatorname{Key}_{I_{1}}\right), \cdots,\left(I_{n}\right.$, Key $\left._{I_{n}}\right)$ & $\begin{array}{l}\text { /* New key is the concatenation of input keys. */ } \\
K e y_{O} \leftarrow K e y_{I_{1}} \cup \cdots \cup K e y_{I_{n}}\end{array}$ \\
\hline Union & $\left(I_{1}, K e y_{I_{1}}\right), \cdots,\left(I_{n}, K e y_{I_{n}}\right)$ & $\begin{array}{l}\text { Let } M: C_{I} \rightarrow C_{O} \text { be the mapping from the columns of input operators to } \\
\text { columns of } O \text {. } \\
\text { Key }_{O} \leftarrow \bigcup_{I_{i} \in I}\left(\bigcup_{c \in K_{\text {Key }} I_{i}} M(c)\right)\end{array}$ \\
\hline GroupBy & $(I$, KeyI $)$ & $\mathrm{Key}_{O} \leftarrow$ the grouping columns of $O$. \\
\hline Table & - & $\mathrm{Key}_{O} \leftarrow$ the primary key of $O$. \\
\hline
\end{tabular}

Table 3. Deriving canonical keys for XQGM operators.

\begin{tabular}{|c|c|c|}
\hline Operator type & Output event & Input (operator,event) pairs \\
\hline \multirow{3}{*}{ Select, Project } & $\operatorname{Delete}(O)$ & $\begin{array}{l}\operatorname{DELETE}(I)(I \text { is the input operator); } \\
\operatorname{UpDate}\left(I, C_{\sigma}\right) \text { where } C_{\sigma} \text { are the columns used in the selection condition }\end{array}$ \\
\hline & $\operatorname{INSERT}(O)$ & $\begin{array}{l}\operatorname{INSERT}(I) \\
\operatorname{UPDATE}\left(I, C_{\sigma}\right)\end{array}$ \\
\hline & UPDATE $(O, C)$ & $\operatorname{UPDATE}(I, C)$ \\
\hline \multirow{3}{*}{ Join } & $\operatorname{Delete}(O)$ & $\begin{array}{l}\text { DELETE }(I) \text { for any input operator } I \\
\text { UPDATE }(I, C) \text {, where } C_{I} \text { are the columns of operator } I \text {. }\end{array}$ \\
\hline & $\operatorname{INSERT}(O)$ & $\begin{array}{l}\operatorname{INSERT}(I) \text { for any input operator } I \text {; } \\
\operatorname{UPDATE}(I, C)\end{array}$ \\
\hline & $\operatorname{UPDATE}(O, C)$ & $\operatorname{UPDATE}\left(I, C_{I}\right)$ \\
\hline \multirow{3}{*}{ GroupBy } & $\operatorname{Delete}(O)$ & $\begin{array}{l}\operatorname{DeLETE}(I) ; \\
\operatorname{UpDATE}(I, G) \text {, where } G \text { is the set of grouping columns }\end{array}$ \\
\hline & $\operatorname{INSERT}(O)$ & $\begin{array}{l}\operatorname{INSERT}(I) ; \\
\operatorname{UpDATE}(I, G)\end{array}$ \\
\hline & $\operatorname{UpdATE}(O, C)$ & $\begin{array}{l}\text { UPdATE }(I, C) \text {; } \\
\text { INSERT }(I) \text { unless } C \subseteq G \text {; } \\
\text { DeLETE }(I) \text { unless } C \subseteq G\end{array}$ \\
\hline \multirow{3}{*}{ Union } & $\operatorname{Delete}(O)$ & $\begin{array}{l}\operatorname{DELETE}(I) \text { for any input operator } I \\
\text { UPDATE }\left(I, I_{C}\right) \text { for any input operator } I \text { (Note that } \operatorname{DELETE}(O) \text { could be } \\
\text { caused by an UPDATE where a previously unique tuple becomes a duplicate.) }\end{array}$ \\
\hline & $\operatorname{INSERT}(O)$ & $\begin{array}{l}\operatorname{INSERT}(I) \text { for any input operator } I \text {; } \\
\operatorname{UpDATE}\left(I, I_{C}\right) \text { for any input operator } I \text { (analogously to } \operatorname{DeLETE}(O) \text { ) }\end{array}$ \\
\hline & UPDATE $(O, C)$ & $\operatorname{UPDATE}\left(I, I_{C}\right)$ for any input operator $I$ \\
\hline
\end{tabular}

Table 4. Operator-specific rules used in event pushdown. 


\begin{tabular}{|c|c|c|}
\hline$[31]\langle$ MainModule $\rangle$ & $\longrightarrow$ & $\langle$ Prolog $\rangle\langle$ QueryBody $\rangle$ \\
\hline$\langle$ MainModule $*\rangle$ & $\longrightarrow$ & $\langle$ QueryBody $\rangle$ \\
\hline$[72]\langle$ AxisStep $\rangle$ & $\longrightarrow$ & $\begin{array}{l}(\langle\text { ForwardStep }\rangle \mid\langle\text { ReverseStep }\rangle) \\
\mid\langle\text { Predicates }\rangle\end{array}$ \\
\hline$\langle$ AxisStep $*\rangle$ & $\longrightarrow$ & $\langle$ ForwardStep $\rangle\langle$ Predicates $\rangle$ \\
\hline$[89]\langle$ ForwardAxis $\rangle$ & $\longrightarrow$ & 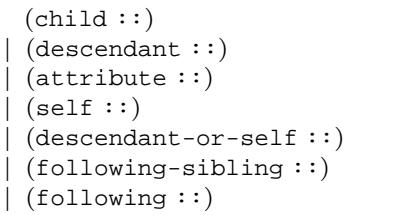 \\
\hline$\langle$ ForwardAxis $*\rangle$ & $\longrightarrow$ & 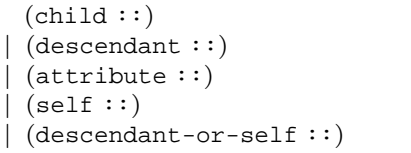 \\
\hline$[56]\langle$ AndExpr $\rangle$ & $\longrightarrow$ & $\begin{array}{l}\langle\text { InstanceofExpr }\rangle \\
\{\text { and }\langle\text { InstanceofExpr }\rangle\}\end{array}$ \\
\hline$\langle$ AndExpr $*\rangle$ & $\longrightarrow$ & $\begin{array}{l}\langle\text { ComparisonExpr }\rangle \\
\{\text { and }\langle\text { ComparisonExpr }\rangle\}\end{array}$ \\
\hline
\end{tabular}

Figure 20. Supported XQuery grammar. Nonterminals marked with an asterisk $(*)$ indicate our implementation, while the bracketed number refers to the rule in the XQuery specification [27].

\section{E.1. Avoiding Spurious UPDATE Events}

The goal of CreateANGraph is to produce an XQGM graph which, when evaluated, will produce the values of OLD_NODE and NEW_NODE for each XML node which was inserted, updated, or deleted. If, as we shall prove, CreateAKGraph (Figure 8) is correct, then the anti-join at the top of $G_{\text {affected }}$ (lines 13 and 15 of CreateANGraph) prevents INSERT and DELETE triggers from producing spurious output.

However, when the XML trigger is on an UPDATE event, there are cases in which spurious updates might be detected. To see why, suppose the view for our running example (Figure 3) were changed so that instead of producing the list of vendors for each product, it only produced the minimum price for each. The XQGM graph for this modified view is shown in Figure 21. Note that the only difference from the original (Figure 5) is in the Project and GroupBy (boxes 4 and 5).

Now suppose vendor initially contains the following two tuples:

\begin{tabular}{|c|c|c|}
\hline vid & pid & price \\
\hline Amazon & P1 & 100.00 \\
Buy.com & P1 & 60.00 \\
\hline
\end{tabular}

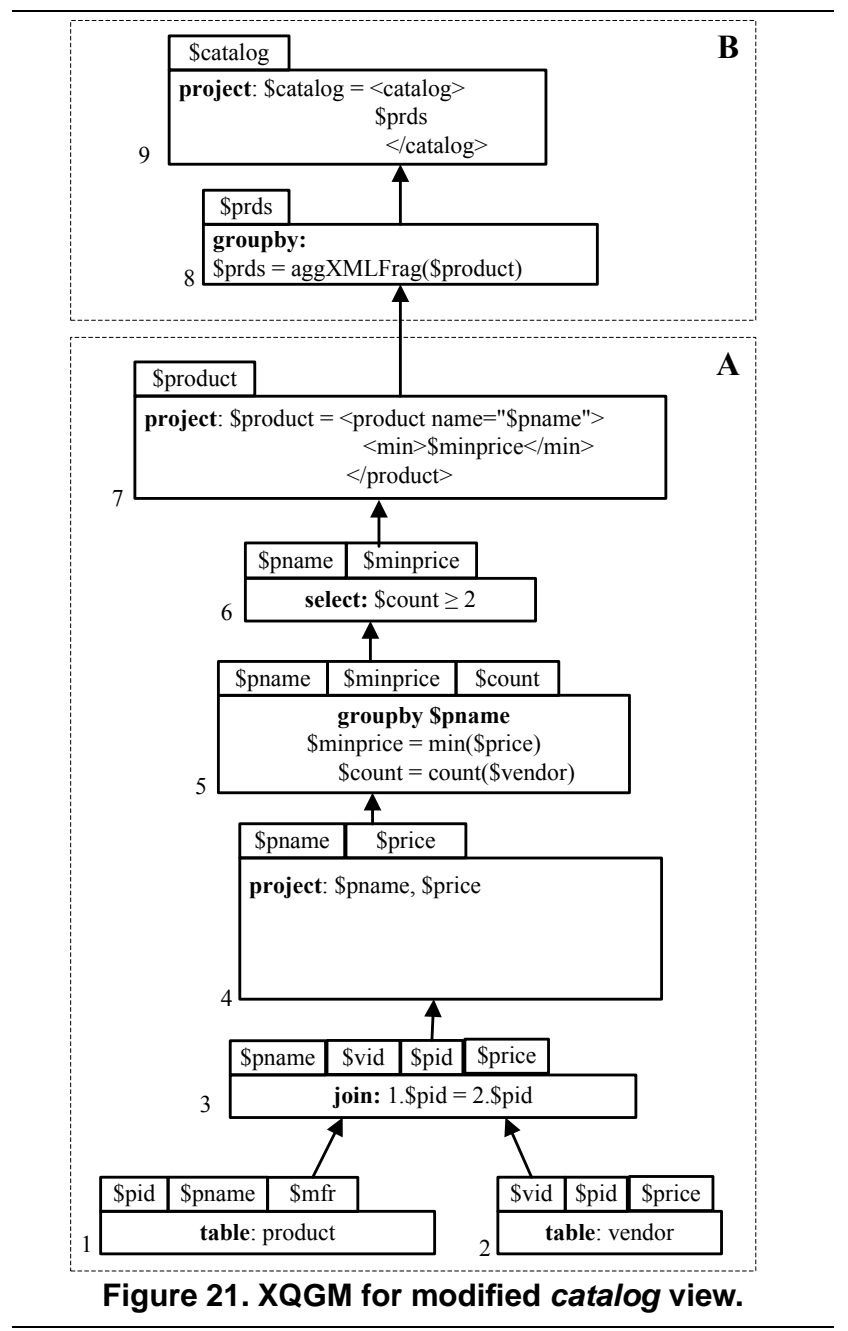

and one vendor updates its price:

\begin{tabular}{|c|c|c|}
\hline \multicolumn{1}{c}{$\nabla$ vendor } & \multicolumn{3}{c|}{$\Delta$ vendor } \\
\hline vid & pid & price \\
\hline Amazon & $\mathrm{P} 1$ & 100.00 \\
\hline
\end{tabular}$\quad$\begin{tabular}{|c|c|c|}
\hline vid & pid & price \\
\hline Amazon & $\mathrm{P} 1$ & 75.00 \\
\hline
\end{tabular}

Since the view creates product elements, P 1 will be identified by our algorithm as an affected key because $\triangle$ vendor and $\nabla$ vendor (potentially) affect the aggregate produced by the GroupBy operator in Box 5. However, since this particular update does not change the minimum price, the XML node for product $\mathrm{P} 1$ remains unchanged: both before and after the update, the node is simply:

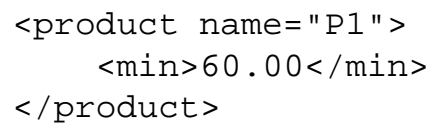

On the other hand, if the new price for Amazon had been, say, \$50.00, this XML node would have been affected. Thus, when the XML trigger is on an UPDATE event, we need to ensure that the node in question was actually updated. The simplest, but far from 
the most efficient, solution is to place a selection condition at the top of $G_{\text {affected }}$ (Line 11 in Figure 12) which filters out those (OLD_NODE, NEW_NODE) pairs where OLD_NODE = NEW_NODE. This is implemented as a string comparison in the tagger (since it's a comparison of the full XML nodes), which has two drawbacks. First, it is expensive when the nodes are large. Second, and more importantly, it requires passing the entire (OLD_NODE, NEW_NODE) pair to the middleware, which prevents many of the optimizations which would otherwise be performed by XQGM graph rewrite rules when the Condition and Action do not require the entire nodes. We therefore present some optimizations and prove their correctness in Appendix F. For this section, however, we assume the use of the simpler, less-efficient approach.

\section{E.2. Proof of Correctness of CreateAKGraph}

Central to our proof of Theorem 2 is the correctness of the affected-keys algorithm, CreateAKGraph (Figure 8).

First, we formally define some terminology. In the following, we use $R(T, D)$ to denote the contents of table $T$ in database state $D$. (In other words, $R(T, D)=R(o, D)$ where $o$ is the XQGM operator Table $(T)$.)

Definition 5 (Valid transitional tables). For any given single-table database transition $D \stackrel{T}{\rightarrow} D^{\prime},(\nabla T, \triangle T)$ is a valid pair of transitional tables iff

$$
\begin{gathered}
\nabla T \subseteq R(T, D), \triangle T \subseteq R\left(T, D^{\prime}\right), \\
\nabla T \supseteq\left\{x \mid x \in R(T, D) \wedge x \notin R\left(T, D^{\prime}\right)\right\}, \\
\triangle T \supseteq\left\{x \mid x \in R\left(T, D^{\prime}\right) \wedge x \notin R(T, D)\right\}, \\
\text { and }(R(T, D)-\nabla T)=R\left(T, D^{\prime}\right)-\triangle T .
\end{gathered}
$$

Definition 6 (Hypothetical state). For a given database state $D$ and a transitional table $d T$, the hypothetical state $D_{-d T}$ is the database state such that $R\left(T, D_{-d T}\right)=R(T, D)-d T$ and for all tables $T^{\prime} \neq T, R\left(T^{\prime}, D_{-d T}\right)=R\left(T^{\prime}, D\right)$.

We refer to a database transition as monotonic if $\nabla T=\varnothing$ or $\triangle T=\varnothing$. It follows from Definitions 5 and 6 that both $D \stackrel{T}{\rightarrow} D_{-\triangle T}^{\prime}$ and $D_{-\triangle T}^{\prime} \stackrel{T}{\rightarrow} D^{\prime}$ are monotonic transitions.

Definition 7 ("Affected"). A tuple $t$ is said to be affected in view $G$ by relational transition $D \stackrel{*}{\rightarrow} D^{\prime}$ iff $t$ is updated, inserted, or deleted in $G$ by $D \stackrel{*}{\rightarrow} D^{\prime}$ (as per Definitions 2 and 3).

We now prove the correctness of CreateAKGraph.

Lemma 1 (Correctness of CreateAKGraph). Given a view graph $G$, a relational table $T$, and a monotonic database transition $D_{1} \stackrel{T}{\rightarrow} D_{2}$ with non-empty transition table $d T$, let $O^{\prime}=$ CreateAKGraph $\left(o_{G}, T, d T\right)$.
Then $c k v_{O^{\prime}}(x) \in R\left(O^{\prime}, D_{2}\right)$ for all tuples $x$ where $x$ is affected in $G$ by $D_{1} \stackrel{T}{\rightarrow} D_{2}$.

Proof. We prove Lemma 1 by induction on the depth of $G$.

Base case: depth $=1$.

In this case, the view graph only consists of a single operator, Table $(X)$, for some relational table $X$.

Suppose $T \neq X$. Since we stipulated that a database transition $D_{1} \stackrel{T}{\rightarrow} D_{2}$ occurred, $D_{1}$ and $D_{2}$ are identical states except for the contents of table $T$. Therefore, since $R\left(\operatorname{Table}(X), D_{1}\right)=R\left(\operatorname{Table}(X), D_{2}\right)$, there are no tuples affected, so the lemma is vacuously true.

On the other hand, suppose that $T=X$. Then CreateAKGraph $\left(o_{G}, T, d T\right)=\pi_{T, k e y}(\operatorname{Table}(d T))$. Hence, by the definition of transitional tables, $R\left(O^{\prime}, D_{2}\right)$ contains all tuples $x$ affected by $D_{1} \stackrel{T}{\rightarrow} D_{2}$.

Thus, the base case holds.

Induction Hypothesis: For a graph $H$ of depth $\leq k$, suppose Lemma 1 holds.

We will now show that Lemma 1 holds for a graph $G$ of depth $k+1$. There are four cases, one for each type of operator except for Table (which can only occur at the leaf level of the graph).

\section{Case 1: $o_{G}$ is a GroupBy operator.}

This case is handled by lines 11-18 of the algorithm.

Then there are two cases to consider, depending on the value returned by the recursive call to CreateAKGraph, $I^{\prime}$. First, if $I^{\prime}=\varnothing$, then there are no tuples in the input to the GroupBy which were affected as a result of the database transition. Since the input to the GroupBy is unchanged, and it merely aggregates its input, its output must also be unaffected by the transition; hence, in this case, we simply return $\varnothing$.

Otherwise, the algorithm creates a Join operator, $J$, joining $I$ with $I^{\prime}$, and then returns a new GroupBy operator which merely projects out the values of the grouping columns of $o_{G}$. Note that for a GroupBy operator, the grouping columns are its canonical key. By the induction hypothesis, $c k v_{I^{\prime}}(x) \in R\left(I^{\prime}, D_{2}\right)$ for every tuple $x$ affected by the transition. If $c k v_{I^{\prime}}(x)$ is produced by $I^{\prime}$, then $J$ will produce all tuples $y$ where $c k v_{O_{G}}(x)=c k v_{O_{G}}(y)$, and $O^{\prime}$ will produce $c k v_{O^{\prime}}(y)$. In other words, for each tuple produced by operator $I$ affected by the transition, $O^{\prime}$ produces the corresponding value of the canonical key of the GroupBy operator, $O^{\prime}$.

Finally, since each tuple $z$ produced by a GroupBy operator $Q$ depends only on those input tuples $w$ where $c k v_{Q}(w)=c k v_{Q}(z)$, the keys of all tuples affected in $G$ by the transition $D_{1} \stackrel{T}{\rightarrow} D_{2}$ are included in $R\left(O^{\prime}, D_{2}\right)$. 


\section{Case 2: $o_{G}$ is a Select or Project operator.}

This case is handled by lines 20-21 of the algorithm.

Both Select and Project take a single input, I. Suppose, by contradiction, that there exists a tuple $x$ such that $x$ is affected in $G$ by $D_{1} \stackrel{T}{\rightarrow} D_{2}$, but $c k v_{O^{\prime}}(x) \notin R\left(O^{\prime}, D_{2}\right)$. By the algorithm (line 21), $O^{\prime}=I^{\prime}$, so it must also be true that $c k v_{I^{\prime}}(x) \notin R\left(I^{\prime}, D_{2}\right)$. Let $y$ be the tuple produced by $I$ such that $c k v_{I}(y)=c k v_{I}(x)$. There must be exactly one such tuple, because Project does not change the cardinality of its input, and Select can only decrease it. Therefore $c k v_{I^{\prime}}(y) \notin R\left(I^{\prime}, D_{2}\right)$. By the induction hypothesis, this implies that $y$ is not affected by $D_{1} \stackrel{T}{\rightarrow} D_{2}$, for if it were, $c k v_{I^{\prime}}(y)$ would be in $R\left(I^{\prime}, D_{2}\right)$. Both Select and Project are deterministic (given the limitations on XQuery functions laid out in Appendix D), and compute each output tuple in terms of exactly one input tuple. But this yields a contradiction, because an unaffected input tuple $y$ resulted in an affected corresponding output tuple $x$.

\section{Case 3: $o_{G}$ is a Join.}

This case is handled by lines 24-39 of the algorithm.

A Join with predicates is semantically equivalent to a Join with no predicates (i.e., a cross-product) followed by a Select imposing the predicates. Since a Select requires no additional operators added to the affected-keys graph (see Case 2 above), we can assume, without loss of generality, that $o_{G}$ has no predicates.

Furthermore, a Join with fewer than 2 input operators is equivalent to a Select, and a Join with more than 2 input operators can be split up into several joins, i.e., $\operatorname{Join}\left(I_{1}, I_{2}, \cdots, I_{n}\right)=\operatorname{Join}\left(I_{1}, \operatorname{Join}\left(I_{2}, \cdots, I_{n}\right)\right)$. We therefore make the additional simplifying assumption that each Join has exactly 2 inputs.

The algorithm begins by invoking CreateAKGraph recursively on each of the inputs $\left(I_{0}\right.$ and $\left.I_{1}\right)$. There are three possible cases to consider: first, suppose both invocations return $\varnothing$. By the induction hypothesis, this implies that the input to $o_{G}$ is unchanged; since Join is deterministic, its output must also be unchanged.

The second possibility is that CreateAKGraph returned $\varnothing$ for exactly one of $I_{0}$ or $I_{1}$, and returned some $I^{\prime}$ for the other. The proof for this case is almost identical to the proofby-contradiction used in Case 2 for Select, so we just sketch it out: assume that there exists a tuple $x$ such that $x$ is affected in $G$ by $D_{1} \stackrel{T}{\rightarrow} D_{2}$, but $c k v_{O^{\prime}}(x) \notin R\left(O^{\prime}, D_{2}\right)$; then a contradiction arises because only one leg of the Join changed, and $I^{\prime}=O^{\prime}$, so the corresponding tuple $y \in R\left(I^{\prime}, D_{2}\right)$ should have been identified by $I^{\prime}$.

The third and final possibility is that CreateAKGraph returned $I_{0}^{\prime}$ and $I_{1}^{\prime}$ for $I_{0}$ and $I_{1}$, respectively. In this case, we produce $O^{\prime}$ by creating two Join operators- $J_{a}$ computing the cross-product $\left(I_{0}^{\prime} \times I_{1}\right)$, and $J_{b}$ computing $\left(I_{0} \times I_{1}^{\prime}\right)$ - and taking their Union. Suppose by contradic- tion that there exists a tuple $x$ such that $x$ is affected in $G$ by $D_{1} \stackrel{T}{\rightarrow} D_{2}$, but $c k v_{O^{\prime}}(x) \notin R\left(O^{\prime}, D_{2}\right)$. Then, by definition of a cross-product, there exists exactly one pair of tuples $(y, z)$ such that $x=y \cdot z$, i.e. $y \in R\left(I_{0}, D_{2}\right), z \in R\left(I_{1}, D_{2}\right)$, $c k v_{I_{0}}(y)=c k v_{I_{0}}(x)$, and $c k v_{I_{1}}(z)=c k v_{I_{1}}(x)$. Since $x$ was affected, and Join is deterministic, it must be the case that either $y$ or $z$ was also affected. Without loss of generality, assume it was $y$. Then, by the induction hypothesis, it must be the case that $c k v_{I_{0}^{\prime}}(y) \in R\left(I_{0}^{\prime}, D_{2}\right)$. Therefore, since $z \in R\left(I_{1}, D_{2}\right)$, there is a tuple $x^{\prime} \in R\left(J_{a}, D_{2}\right)$ such that $c k v_{I_{0}^{\prime}}\left(x^{\prime}\right)=c k v_{I_{0}^{\prime}}(y)$ and $c k v_{I_{1}^{\prime}}\left(x^{\prime}\right)=c k v_{I_{1}^{\prime}}(z)$. But this yields a contradiction, since the Union will simply propagate $x^{\prime}$, and $x^{\prime}=c k v_{O^{\prime}}(x)$, contradicting our original assumption that $c k v_{O^{\prime}}(x) \notin R\left(O^{\prime}, D_{2}\right)$.

\section{Case 4: $o_{G}$ is a Union.}

The final possibility is that $o_{G}$ is a Union operator; this is handled in lines 43-53. The algorithm for Union calls CreateAKGraph recursively on each of its inputs $i$, and then creates a new Union operator computing the union of each of these results $i^{\prime}$.

We prove the correctness for this final case by contradiction. Suppose there exists some tuple $x$ such that $x$ is affected in $G$ by the transition $D_{1} \stackrel{T}{\rightarrow} D_{2}$, but $x \notin R\left(O^{\prime}, D_{2}\right)$. By definition of the Union operator, there must be at least one input operator $I$ such that $x$ was affected in $I$. By the induction hypothesis, $c k v_{I^{\prime}}(x) \in R\left(I^{\prime}, D_{2}\right)$. However, this yields a contradiction because $I^{\prime}$ is one of the inputs to $O^{\prime}$, and the semantics of Union require that $\forall y\left(y \in I^{\prime} \rightarrow\right.$ $y \in O^{\prime}$.

\section{E.3. Proof of Correctness of CreateANGraph}

Before we can prove the correctness of CreateANGraph, we must first prove the following lemma:

Lemma 2. For a database transition $D \stackrel{T}{\rightarrow} D^{\prime}$ with transitional tables $\triangle T$ and $\nabla T$, and a view graph $G$, if a tuple $t$ is affected in $G$ by $D \stackrel{T}{\rightarrow} D^{\prime}$, then either $t$ is affected by $D \stackrel{T}{\rightarrow} D_{-\triangle T}^{\prime}$, or $t$ is affected by $D_{-\triangle T}^{\prime} \stackrel{T}{\rightarrow} D^{\prime}$.

Proof. For conciseness of notation, let $\bar{D}=D_{-\triangle T}^{\prime}$. Suppose by contradiction that (1) $t$ is affected by $D \stackrel{T}{\rightarrow} D^{\prime}$, but (2) $t$ is not affected by $D \stackrel{T}{\rightarrow} \bar{D}$ and $t$ is not affected by $\bar{D} \stackrel{T}{\rightarrow} D^{\prime}$. By Definitions 2 and 3, it follows from (2) that one of two cases is possible: either (3) $\left(t \in R\left(o_{G}, D\right) \wedge t \in\right.$ $\left.R\left(o_{G}, \bar{D}\right) \wedge t \in R\left(o_{G}, D^{\prime}\right)\right)$ or $(4)\left(t \notin R\left(o_{G}, D\right) \wedge t \notin\right.$ $\left.R\left(o_{G}, \bar{D}\right) \wedge t \notin R\left(o_{G}, D^{\prime}\right)\right)$.

If (3) holds, then $t \in R\left(o_{G}, D\right) \wedge t \in R\left(o_{G}, D^{\prime}\right)$, so it follows from Definitions 2 and 3 that $t$ is neither inserted, deleted, or updated in $G$ by $D \stackrel{T}{\rightarrow} D^{\prime}$, contradicting our assumption (1) that $t$ is affected by $D \stackrel{T}{\rightarrow} D^{\prime}$. 
On the other hand, if (4) holds, then $t \notin R\left(o_{G}, D\right) \wedge t \notin$ $R\left(o_{G}, D^{\prime}\right)$; again, it follows from Definitions 2 and 3 that $t$ is neither inserted, deleted, or updated in $G$ by $D \stackrel{T}{\rightarrow} D^{\prime}$, contradicting assumption (1).

We now proceed with the proof of Theorem 2, the correctness of CreateANGraph. Please refer to Figure 12 for the text of the algorithm, which is referenced throughout this proof.

Theorem 2. Given an event $E$, view graph $G$, and table $T$, CreateANGraph $(E, G, T)$ produces graph $G_{\text {affected }}$ such that for all valid database transitions $D \stackrel{T}{\rightarrow} D^{\prime}$, (OLD_NODE, NEW_NODE) $\in R\left(o_{G_{\text {affected }}}, D^{\prime}\right)$ iff:

(a) $E=\operatorname{UPDATE} \wedge$ OLD_NODE $\in R\left(o_{G}, D\right) \wedge$ NEW_NODE $\in$ $R\left(o_{G}, D^{\prime}\right) \wedge c k v_{o_{G}}($ OLD_NODE $)=c k v_{o_{G}}($ NEW_NODE $) \wedge$ $v($ OLD_NODE $) \neq v($ NEW_NODE $)$, or

(b) $E=$ INSERT $\wedge$ OLD_NODE $=\varnothing \wedge$ NEW_NODE $\in$ $R\left(o_{G}, D^{\prime}\right) \wedge \nexists x \mid\left(x \in R\left(o_{G}, D\right) \wedge c k v_{o_{G}}\left(\mathrm{NEW} \_\mathrm{NODE}\right)=\right.$ $\left.c k v_{o_{G}}(x)\right)$, or

(c) $E=$ DELETE $\wedge$ NEW_NODE $=\varnothing \wedge$ OLD_NODE $\in$ $R\left(o_{G}, D\right) \wedge \nexists x \mid\left(x \in R\left(o_{G}, D^{\prime}\right) \wedge c k v_{o_{G}}(\right.$ OLD_NODE $)=$ $\left.c k v_{o_{G}}(x)\right)$.

Proof. Lemma 1 proved that if some tuple $x$ is affected in $G$ by the database transition $D_{-\triangle T}^{\prime} \stackrel{T}{\rightarrow} D^{\prime}$, then $c k v_{o_{G}}(x) \in R\left(\operatorname{CreateAKGraph}\left(o_{G}, T, \triangle T\right), D^{\prime}\right)$, and that if some tuple $x$ is affected in $G$ by $D \stackrel{T}{\rightarrow} D_{-\triangle T}^{\prime}$, then $c k v_{o_{G}}(x) \in R\left(\right.$ CreateAKGraph $\left.\left(o_{G_{\text {old }}}, T_{\text {old }}, \nabla T\right), D^{\prime}\right)$. As Lemma 2 showed, for any tuple $x$ affected in $G$ by $D \stackrel{T}{\rightarrow} D^{\prime}$, it must be affected either by $D_{-\triangle T}^{\prime} \stackrel{T}{\rightarrow} D^{\prime}$ or by $D \stackrel{T}{\rightarrow} D_{-\triangle T}^{\prime}$. Therefore Union created in line 6 produces a superset of affected keys. I.e., for any affected tuple $x$, it must be the case that $x \in R\left(O_{u}, D^{\prime}\right)$ (see line 6).

We now consider each of the three event types separately, and we prove the theorem in both directions for each.

(a) $E=$ UPDATE.

Suppose $E=$ UPDATE, and there exist OLD_NODE and NEW_NODE such that OLD_NODE $\in R\left(o_{G}, D\right) \wedge$ NEW_NODE $\in$ $R\left(o_{G}, D^{\prime}\right) \wedge c k v_{o_{G}}\left(\mathrm{OLD} \_\mathrm{NODE}\right)=c k v_{o_{G}}\left(\mathrm{NEW} \_\mathrm{NODE}\right) \wedge$ $v($ OLD_NODE $) \neq v$ (NEW_NODE). This is the definition of an UPDATE event on $o_{G}$, and since we have shown that $R\left(O_{u}, D^{\prime}\right)$ contains $c k v_{o_{G}}(x)$ for all tuples $x$ affected by the database transition, we can infer that $R\left(O_{u}, D^{\prime}\right)$ contains $c k v_{O_{G}}$ (OLD_NODE). Then, since OLD_NODE $\in R\left(o_{G}, D\right)$, it follows that OLD_NODE $\in R\left(O_{\text {old }}, D^{\prime}\right)$ (line 8); similarly, since NEW_NODE $\in R\left(o_{G}, D^{\prime}\right)$, therefore NEW_NODE $\in R\left(O_{\text {new }}, D^{\prime}\right)$ (line 8). Next, since there is exactly one tuple in each of $R\left(O_{\text {old }}, D^{\prime}\right)$ and
$R\left(O_{\text {new }}, D^{\prime}\right)$, the inner join (line 10) produces exactly (OLD_NODE, NEW_NODE). Since we initially stipulated that OLD_NODE $\neq$ NEW_NODE, the final Select (line 11) does not remove this pair from the output. Therefore, $G_{\text {affected }}$ produces the tuple (OLD_NODE, NEW_NODE).

Conversely, suppose $E=$ UPDATE, and there exists (OLD_NODE, NEW_NODE) $\in R\left(O_{G_{\text {affected }}}, D^{\prime}\right)$. Then, this must have been returned in line 11 , so we can infer that OLD_NODE $\neq$ NEW_NODE. Furthermore, OLD_NODE and NEW_NODE come from $O_{\text {old }}$ and $O_{\text {new }}$, respectively, joined on their respective keys; therefore, since the key of both $O_{\text {old }}$ (line 8) and $O_{\text {new }}$ (line 7) is the same as the key of $G$, we have that $c k v_{O_{G}}\left(\mathrm{OLD} \_\mathrm{NODE}\right)=c k v_{O_{G}}(\mathrm{NEW}$ NODE). Finally, since $R\left(O_{\text {new }}, D^{\prime}\right) \subseteq R\left(G, D^{\prime}\right)$, we can conclude that NEW_NODE $\in R\left(G, D^{\prime}\right)$. We can similarly conclude that OLD_NODE $\in R(G, D)$ because $R\left(O_{\text {old }}, D^{\prime}\right) \subseteq R(G, D)$.

(b) $E=$ INSERT.

Next, suppose that $E=$ INSERT, and there exist OLD_NODE and NEW_NODE such that OLD_NODE = $\varnothing \wedge$ NEW_NODE $\in R\left(o_{G}, D^{\prime}\right) \wedge \nexists x \mid\left(x \in R\left(o_{G}, D\right) \wedge\right.$ $c k v_{O_{G}}(\mathrm{NEW}$ NODE $\left.)=c k v_{o_{G}}(x)\right)$. This is the definition of an INSERT event on $o_{G}$, and since we have shown that $R\left(O_{u}, D^{\prime}\right)$ contains $c k v_{O_{G}}(x)$ for all tuples $x$ affected by the database transition, we can infer that $R\left(O_{u}, D^{\prime}\right)$ contains $c k v_{o_{G}}$ (NEW_NODE). Then, since NEW_NODE $\in R\left(o_{G}, D^{\prime}\right)$, it follows that NEW_NODE $\in R\left(O_{\text {new }}, D^{\prime}\right)$; similarly, since there is no corresponding tuple $x$ in $R\left(o_{G}, D\right)$, it follows that there is no tuple $y \in R\left(O_{\text {old }}, D^{\prime}\right)$ such that $c k v_{o_{G}}(y)=c k v_{O_{G}}$ (NEW_NODE). Therefore, the LeftAntiJoin (line 13) will produce ( $\varnothing$, NEW_NODE).

Conversely, suppose $E=$ INSERT, and there ex-

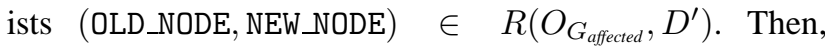
this must have been returned in line 13 , so we can infer that OLD_NODE $=\varnothing$. Furthermore, OLD_NODE and NEW_NODE come from $O_{\text {old }}$ and $O_{\text {new }}$, respectively, antijoined on their respective keys. Therefore, since the key of both $O_{\text {old }}$ (line 8 ) and $O_{\text {new }}$ (line 7) is the same as the key of $G$, we have that NEW_NODE $\in R\left(G, D^{\prime}\right)$, and $\nexists y \mid\left(y \in R\left(o_{O_{\text {old }}}, D^{\prime}\right) \wedge c k v_{o_{G}}(y)=c k v_{o_{G}}(\right.$ NEW_NODE $\left.)\right)$. Because NEW_NODE $\in R\left(O_{\text {new }}, D^{\prime}\right)$, we conclude that $c k v_{O_{G}}(\mathrm{NEW}$ NODE $) \in R\left(O_{u}, D^{\prime}\right)$. Therefore, since we have shown that $O_{\text {old }}$ does not contain a corresponding tuple $y$, it follows that $\nexists x \mid\left(x \in R\left(o_{G}, D\right) \wedge c k v_{o_{G}}\left(\mathrm{NEW} \_\mathrm{NODE}\right)=\right.$ $\left.c k v_{o_{G}}(x)\right)$.

(c) $E=$ DELETE.

The final case is analogous to (b). Suppose that $E=$ DELETE, and there exist OLD_NODE and NEW_NODE such that NEW_NODE $=\varnothing \wedge$ OLD_NODE $\in R\left(o_{G}, D\right) \wedge$ $\nexists x \mid\left(x \in R\left(o_{G}, D^{\prime}\right) \wedge c k v_{o_{G}}(\right.$ OLD_NODE $\left.)=c k v_{o_{G}}(x)\right)$. This is the definition of a DELETE event on $o_{G}$, and since we have shown that $R\left(O_{u}, D^{\prime}\right)$ contains $c k v_{o_{G}}(x)$ for all tuples $x$ affected by the database transition, we 
can infer that $R\left(O_{u}, D^{\prime}\right)$ contains $c k v_{O_{G}}$ (OLD_NODE). Then, since OLD_NODE $\in R\left(o_{G}, D\right)$, it follows that OLD_NODE $\in R\left(O_{\text {old }}, D^{\prime}\right)$; similarly, since there is no corresponding tuple $x$ in $R\left(o_{G}, D^{\prime}\right)$, it follows that there is no tuple $y \in R\left(O_{\text {new }}, D^{\prime}\right)$ such that $c k v_{O_{G}}(y)=c k v_{O_{G}}$ (OLD_NODE). Therefore, the RightAntiJoin (line 15) will produce (OLD_NODE, $\varnothing$ ).

Conversely, suppose $E=$ Delete, and there ex-

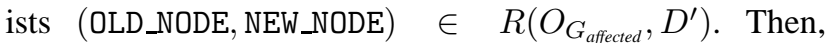
this must have been returned in line 15 , so we can infer that NEW_NODE $=\varnothing$. Furthermore, OLD_NODE and NEW_NODE come from $O_{\text {old }}$ and $O_{\text {new }}$, respectively, antijoined on their respective keys. Therefore, since the key of both $O_{\text {old }}$ (line 8) and $O_{\text {new }}$ (line 7) is the same as the key of $G$, we have that OLD_NODE $\in R(G, D)$, and $\nexists y \mid\left(y \in R\left(o_{O_{\text {new }}}, D^{\prime}\right) \wedge c k v_{o_{G}}(y)=c k v_{O_{G}}(\right.$ OLD_NODE $\left.)\right)$. Because OLD_NODE $\in R\left(O_{\text {old }}, D^{\prime}\right)$, we conclude that $c k v_{O_{G}}$ (OLD_NODE) $\in R\left(O_{u}, D^{\prime}\right)$. Therefore, since we have shown that $O_{\text {new }}$ does not contain a corresponding tuple $y$, it follows that $\nexists x \mid\left(x \in R\left(o_{G}, D^{\prime}\right) \wedge c k v_{o_{G}}\right.$ (OLD_NODE) $=$ $\left.c k v_{o_{G}}(x)\right)$.

\section{F. Optimizations for CreateANGraph}

As described in Appendix E.1, in the CreateANGraph algorithm (Figure 12), we initially put a Select operator (line 11) at the top of the affected-node graph in order to ensure that OLD_NODE and NEW_NODE actually differ; however, doing this comparison in the tagger can be expensive. In this section, we identify a general class of views for which we do not have to explicitly check whether OLD_NODE and NEW_NODE differ, while still ensuring that we do not identify spurious updates - many views, including the running example in the paper, fall into this class. For views that do not fall into this class, we present a few optimizations that can push down the check to the relational engine under certain conditions.

\section{F.1. Definitions}

As Definition 5 shows, the transitional tables provided by the relational database system are actually a superset of the tuples which changed. This is because an update statement such as:

\section{UPDATE VENDOR \\ $\mathrm{SET}$ PRICE $=1$ * PRICE}

will result in the transitional tables containing as many rows as there are vendor rows, even though none of the vendor rows actually changed in value. Therefore, CreateAKGraph would produce keys of XML nodes which were not actually updated, and these would not be identified as spurious until reaching the final Select operator.
This problem can be avoided by replacing all references to $\triangle T$ and $\nabla T$ in the SQL trigger with $\triangle T^{\prime}$ and $\nabla T^{\prime}$, respectively, where $\triangle T^{\prime}=\triangle T-\nabla T$ and $\nabla T^{\prime}=\nabla T-\triangle T$. Then we can refine Definition 5:

Definition 8 (Pruned transitional tables). For any given single-table database transition $D \stackrel{T}{\rightarrow} D^{\prime}$, the pruned transition tables $\mathbf{\Delta} T$ and $\boldsymbol{\nabla} T$ are:

$$
\begin{gathered}
\Delta T=\left\{x \mid x \in R\left(T, D^{\prime}\right) \wedge x \notin R(T, D)\right\}, \text { and } \\
\boldsymbol{\nabla} T=\left\{x \mid x \in R(T, D) \wedge x \notin R\left(T, D^{\prime}\right)\right\} .
\end{gathered}
$$

For a large class of views, including the running example in the paper, we can actually remove the selection condition OLD_NODE $\neq$ NEW_NODE from CreateANGraph if we prune the transition tables. This class of views, which we call injective, has the property that there is a one-to-one mapping between each XML node produced by $o_{G}$ (the top operator of the view graph) and the set of relational tuples used to construct the node.

In order to prove this claim, we must first define the notion of an injective view more formally. We begin by defining the contributing set of a tuple: intuitively, for any tuple $t$ produced by an operator $o$, there is a set of tuples produced by each of its input operators $o_{i}$ which contributes to $t$. For example, the contributing set of a tuple $t$ produced by a GroupBy operator is the set of all input tuples having the same grouping-column value as $t$. For a Project or Select, the contributing set of a tuple $t$ is the input tuple from which $t$ is computed by projection or selection, respectively. We now formalize this notion of a contributing set of a tuple for arbitrary operators.

Definition 9 (Contributing set). Given an operator $(o)$, one of its input operators $\left(o_{i}\right)$, a database state $(D)$, and a tuple $\left(t_{o} \in R(o, D)\right)$, the contributing set of $t_{o}$ is:

$$
\begin{aligned}
& \zeta\left(t_{o}, o_{i}, o, D\right)=\left\{t_{i} \in R\left(o_{i}, D\right) \mid\right. \\
& \forall D^{\prime}\left(t_{i} \in R\left(o_{i}, D^{\prime}\right) \rightarrow\right. \\
& \left.\left.\quad \exists t_{o}^{\prime}\left(t_{o}^{\prime} \in R\left(o, D^{\prime}\right) \wedge c k v_{o}\left(t_{o}\right)=c k v_{o}\left(t_{o}^{\prime}\right)\right)\right)\right\} .
\end{aligned}
$$

In the following, $\tilde{C}(o)$ is the set of columns produced by operator $o$. For a tuple $t$ produced by operator $o$, we denote the value of columns $C$ (where $C \subseteq \tilde{C}(o)$ ) as $\pi_{C}(t)$. We similarly denote projection for a set of tuples: $\pi_{C}(S)=\left\{\pi_{C}(t) \mid t \in S\right\}$. Finally, when $C$ is a set of columns belonging to (potentially) multiple operators, then $(C \mid O)$ denotes the subset of $C$ belonging to operator $o$; i.e. $(C \mid o)=C \cap \tilde{C}(o)$.

We now define injection for a single operator in terms of the contributing set of each tuple it produces:

Definition 10 (Injection for operators). Given an XQGM operator (o) with a set of input operators $(I)$, a set of o's columns $\left(C_{o}\right)$, and a subset of I's columns $\left(C_{I}\right)$ : the columns $C_{o}$ are injective with respect to the columns $C_{I}$ (denoted as $C_{I} \mapsto C_{o}$ ) iff: 


$$
\begin{gathered}
\forall t_{1}, D_{1}, t_{2}, D_{2}( \\
\left(t_{1} \in R\left(o, D_{1}\right) \wedge t_{2} \in R\left(o, D_{2}\right) \wedge \pi_{C_{o}}\left(t_{1}\right)=\pi_{C_{o}}\left(t_{2}\right)\right) \\
\rightarrow\left(\forall o _ { i } \in I \left(\pi_{\left(C_{I} \mid o_{i}\right)}\left(\zeta\left(t_{1}, o_{i}, o, D_{1}\right)\right)=\right.\right. \\
\left.\left.\left.\pi_{\left(C_{I} \mid o_{i}\right)}\left(\zeta\left(t_{2}, o_{i}, o, D_{2}\right)\right)\right)\right)\right) .
\end{gathered}
$$

In other words, if $C_{I} \mapsto C_{o}$, then there is a one-to-one mapping such that for each tuple $t$ produced by that operator, $\pi_{C_{o}}(t)$ (the value of columns $C_{o}$ in tuple $t$ ) maps to a unique set of $C_{I}$ values produced by the input operator(s) $I$.

Definition 11 (Transitive injection). An operator o is transitively injective for $C_{o}$ with respect to a table $T$ (denoted $T \stackrel{*}{\longmapsto} C_{o}$ ) iff one of the following holds:

- $o=\operatorname{Table}(T)$ and $C_{o}$ is all of T's columns, or

- $\exists C_{I}\left(\left(C_{I} \mapsto C_{o}\right) \wedge \forall o_{i}\left(\left(o_{i} \in I\right) \rightarrow\left(T \stackrel{*}{\longmapsto}\left(C_{I} \mid o_{i}\right)\right)\right)\right)$.

That is, an operator is transitively injective for a subset of its output columns, $C_{o}$, if and only if there is a one-to-one mapping such that for every tuple $t$ produced by the operator Table $(T), v_{C_{o}}(t)$ maps to a unique set of tuples in $\operatorname{Table}(T)$.

Finally, we say that a view with graph $G$ is injective for $C$ with respect to table $T$ if its top operator, $o_{G}$, is transitively injective for $C$ with respect to $T$. Although these conditions may seem restrictive, most XML views of relational data are injective with respect to each of their base tables. For example, the original catalog view (Figure 5) is injective with respect to both product and vendor.

In Section F.3, we will prove the following theorem, stating that for injective views, CreateANGraph will not produce spurious updates if we remove the final selection condition in line 11; we refer to this modified version as CreateANOpt.

Theorem 3. Given an UPDATE event, a view graph $G$ which is injective for all columns of its top operator, and table $T$, let $G_{\text {affected }}=$ CreateANGraph $(\operatorname{UPDATE}, G, T)$, and $G_{\text {opt }}=$ CreateANOpt(UPDATE, $\left.G, T\right)$. Then for all valid database transitions $D \stackrel{T}{\rightarrow} D^{\prime}$ with pruned transition tables $\boldsymbol{\Delta} T$ and $\boldsymbol{\nabla} T$, (OLD_NODE, NEW_NODE) $\in R\left(o_{G_{\text {opt }}}, D^{\prime}\right)$ if and only if (OLD_NODE, NEW_NODE) $\in R\left(o_{G_{\text {affected }}}, D^{\prime}\right)$.

\section{F.2. Sufficient Conditions for Injection}

For each operator $o$ in a graph $G$, and a set of columns $C_{o}$, we can determine whether $o$ is injective for $C_{i} \mapsto C_{o}$, based on the type of operator:

- Project, Select, and Join. $o$ is injective for $C_{I} \mapsto C_{o}$ if, for input operator(s) $I$, there exists $C_{I}$ such that for each $c_{i} \in C_{I}$, one of the following holds:

- $c_{i} \in C_{o}$, or

- If $o$ is Project or Select: $\exists c \in C_{o}$ such that $c$ is produced by an injective function which takes $c_{i}$ as a parameter. The most commonly-used such function is the XML constructor function.

- GroupBy. $o$ is injective for $C_{i} \mapsto C_{o}$ if, for its input operator $i$, there exists $C_{i}$ such that for each $c_{i} \in C_{i}$, one of the following holds:

$$
\begin{aligned}
& \text { - } c_{i} \in C_{o} \text {, or } \\
& \text { - } \exists c \in C_{o} \text { such that } c=\operatorname{aggXMLFrag}\left(c_{i}\right) .
\end{aligned}
$$

It is easy to see that the view in Figure 5 satisfies the above conditions. Note that the above conditions are sufficient but not necessary for injection.

\section{F.3. Correctness of Theorem 3}

Given an injective view, can now prove a stronger version of Lemma 1:

Lemma 3. Given a relational table $T$, a view graph $G$ which is injective for columns $C$ w.r.t. $T$, and a monotonic database transition $D_{1} \stackrel{T}{\rightarrow} D_{2}$ with pruned nonempty transitional table $d T$, let $O^{\prime}=$ CreateAKGraph $\left(o_{G}, T, d T\right)$. Then $\nexists x, y, z$ $\left(x \in R\left(O^{\prime}, D_{2}\right) \wedge y \in R\left(o_{G}, D_{1}\right) \wedge c k v_{o_{G}}(y)=x \wedge\right.$ $\left.v_{C}(y)=v_{C}(z) \wedge z \in R\left(o_{G}, D_{2}\right) \wedge c k v_{o_{G}}(z)=x\right)$.

Proof. We prove Lemma 3 by induction on the depth of $G$.

Base case: depth $=1$.

In this case, the view graph only consists of a single operator, Table $(X)$, for some relational table $X$.

Suppose $X \neq T$. Then CreateAKGraph returns $\varnothing$, so there are no tuples $x \in R\left(O^{\prime}, D_{2}\right)$; the lemma is vacuously true.

Otherwise, $X=T$. Then CreateAKGraph $\left(o_{G}, T, d T\right)$ returns $\pi_{\text {T.key }}($ Table $(d T))$. Suppose, by contradiction, that there exist $x, y, z$ contradicting Lemma 3 . By the definition of transitive injection, $C$ must be the set of all columns of $T$; therefore, since $v_{C}(y)=v_{C}(z)$, we infer that $y=z$. The pruned transitional table $d T$ is either $\boldsymbol{\Delta} T$ or $\boldsymbol{\nabla} T$. By Definition 8, if $d T=\mathbf{\Delta} T$, then $z \in R\left(T, D_{2}\right)$ implies that $z \notin R\left(T, D_{1}\right)$; otherwise $d T=\boldsymbol{\nabla} T$ and $y \in R\left(T, D_{1}\right)$ implies that $y \notin R\left(T, D_{2}\right)$. In both cases, a contradiction is reached because we had concluded that $y=z$.

Thus, the base case holds.

Induction Hypothesis: For a graph $H$ of depth $\leq k$, suppose Lemma 3 holds.

We will now show that Lemma 3 holds for a graph $G$ of depth $k+1$. There are four cases, one for each type of operator except for Table (which can only occur at the leaf level of the graph).

Case 1: $o_{G}$ is a GroupBy operator.

Suppose, by contradiction, that there exist tuples $x, y, z$ contradicting Lemma 3. We know that $o_{G}$ is injective for 
$C_{I} \mapsto C$, where $C_{I}$ is some set of columns of the input operator, $I$. By the definition of injection, we know that the set of $I$-tuples used for computing $v_{C}(y)$ and $v_{C}(z)$ did not change in the transition: $\pi_{C_{I}}\left(\zeta\left(y, I, o_{G}, D_{1}\right)\right)=$ $\pi_{C_{I}}\left(\zeta\left(z, I, o_{G}, D_{2}\right)\right)$. Let $Z=\zeta\left(z, I, o_{G}, D_{2}\right)$ and $X_{I}=$ $\left\{c k v_{I}(z) \mid z \in Z\right\}$. Finally, let $C_{g}$ be the grouping columns of $o_{G}$ (note that the set of grouping columns defines the key of a GroupBy operator).

By the induction hypothesis, when CreateAKGraph is invoked recursively (line 11$), R\left(I^{\prime}, D_{2}\right)$ will not contain any $x_{I} \in X_{I}$. Therefore, the Join created in line 15 will not propagate any $z \in Z$. Furthermore, there can't be a tuple $z^{\prime} \notin \zeta\left(z, I, o_{G}, D_{2}\right)$ such that $\pi_{C_{g}}\left(z^{\prime}\right)=x$, because $x=\pi_{C_{g}}(z)$ and therefore $\pi_{C_{g}}\left(z^{\prime}\right)$ would have to be in the contributing set $Z$ (by Definition 9). As a result, since the Join is not propagating any tuples $t$ such that $\pi_{C_{g}}(t)=x$, the GroupBy created in line 17 will not produce $x$. This yields a contradiction, however, since our original assumption was that $x \in R\left(O^{\prime}, D_{2}\right)$.

\section{Case 2: $o_{G}$ is a Select or Project operator.}

Suppose, by contradiction, that there exist tuples $x, y, z$ contradicting Lemma 3. We know that $o_{G}$ is injective for $C_{I} \mapsto C$, where $C_{I}$ is some set of columns of the input operator, $I$. The canonical key for Select and Project is defined to be the same as the key of its input, so there is exactly one pair of input tuples $y^{\prime}, z^{\prime}$ such that $y^{\prime} \in R\left(I, D_{1}\right), z^{\prime} \in$ $R\left(I, D_{2}\right), c k v_{I}\left(y^{\prime}\right)=c k v_{o_{G}}(y)$, and $c k v_{I}\left(z^{\prime}\right)=c k v_{o_{G}}(z)$. By the definition of injection, we know that $v_{C}(y)=v_{C}(z)$ implies that $v_{C_{I}}\left(y^{\prime}\right)=v_{C_{I}}\left(z^{\prime}\right)$.

Therefore, by the induction hypothesis, when CreateAKGraph is invoked recursively (line 20), $R\left(I^{\prime}, D_{2}\right)$ will not contain $x$. However, this yields a contradiction, since $O^{\prime}=I^{\prime}$ (line 21), and our original assumption is that $x \in R\left(O^{\prime}, D_{2}\right)$.

\section{Case 3: $o_{G}$ is a Join.}

If CreateAKGraph returned $\varnothing$ (line 32), then there are no tuples $x \in R\left(O^{\prime}, D_{2}\right)$, so the lemma is vacuously true.

If the recursive call to CreateAKGraph only returned non- $\varnothing$ for a single leg of the Join, then this call will simply return $I^{\prime}$ (line 34 ), and therefore the proof is identical to Case 2.

Thus, we only need consider the case where $O^{\prime}$ is a union of cross-products (lines 36-39). Suppose, by contradiction, that there exist tuples $x, y, z$ contradicting Lemma 3 . We know that $o_{G}$ is injective for $C_{I} \mapsto C$, where $C_{I}$ is a set of columns of the input operators $I$. Since the canonical key of Join is the concatenation of the keys of $I$, we infer that for each $i \in I$, there is exactly one triple of input tuples $\left(x_{i}^{\prime}, y_{i}^{\prime}, z_{i}^{\prime}\right)$ such that $y_{i}^{\prime} \in R\left(i, D_{1}\right), z_{i}^{\prime} \in R\left(i, D_{2}\right)$, $x^{\prime}=c k v_{i}\left(y^{\prime}\right)=c k v_{i}(y)$, and $x^{\prime}=c k v_{i}\left(z^{\prime}\right)=c k v_{i}(z)$. By the definition of injection, we know that $v_{C}(y)=v_{C}(z)$ implies that $v_{C_{i}}\left(y_{i}^{\prime}\right)=v_{C_{i}}\left(z_{i}^{\prime}\right)$.
By the induction hypothesis, when CreateAKGraph is invoked recursively (line 25$), R\left(i^{\prime}, D_{2}\right)$ will not contain $x_{i}^{\prime}$. Therefore, the cross-product $J_{a}=I_{0}^{\prime} \times I_{1}$ (line 37) cannot contain $x$, since $x$ is the concatenation of $x_{0}^{\prime}$ and $x_{1}^{\prime}$, and we have just shown that $x_{0}^{\prime} \notin R\left(I_{0}^{\prime}, D_{2}\right)$. By the same argument, $J_{b}$ (line 38 ) also cannot contain $x$, as $x_{1}^{\prime} \notin R\left(I_{1}^{\prime}, D_{2}\right)$. Therefore, by the semantics of the Union operator, the union of $J_{a}$ and $J_{b}$ (line 39) will not produce $x$. Thus, we have reached a contradiction, since our original assumption was that $x \in R\left(O^{\prime}, D_{2}\right)$.

\section{Case 4: $o_{G}$ is a Union.}

Suppose, by contradiction, that there exist tuples $x, y, z$ contradicting Lemma 3. Then by the definition of the Union operator, $\exists I_{i} \in I$ such that $y \in R\left(I_{i}, D_{1}\right)$, and $\exists I_{j} \in I$ such that $z \in R\left(I_{j}, D_{2}\right)$. Because $o_{G}$ is transitively injective for $C$, it follows that $I_{i}$ and $I_{j}$ are both transitively injective for $C$.

If it were the case that $z \in R\left(I_{i}, D_{2}\right)$, this would contradict the induction hypothesis for $I_{i}$. Therefore, it must be the case that $z \notin R\left(I_{i}, D_{2}\right)$. Because we had stipulated that $o_{G}$ is injective for $c_{I} \mapsto C$, and $y \in$ $R\left(o_{G}, D_{1}\right) \wedge z \in R\left(o_{G}, D_{2}\right)$, it follows from Definition 10 that $\pi_{C}\left(\zeta\left(y, I_{i}, o_{G}, D_{1}\right)\right)=\pi_{C} \zeta\left(\left(z, I_{i}, o_{G}, D_{2}\right)\right)$. Because $c k v_{o_{G}}(y)=c k v_{o_{G}}(z)$, therefore $\left.\pi_{C} \zeta\left(y, I_{i}, o_{G}, D_{1}\right)\right)=$ $\pi_{C}\left(\zeta\left(z, I_{i}, o_{G}, D_{1}\right)\right)$. However, this yields a contradiction because $z \in \pi_{C}\left(\zeta\left(z, I_{i}, o_{G}, D_{1}\right)\right)$ but $z \notin \pi_{C}\left(\zeta\left(z, I_{i}, o_{G}, D_{2}\right)\right)$.

We have now proven that for a single transition, CreateAKGraph will not produce the keys of any XML nodes whose value did not actually change. However, this is not sufficient, as CreateAKGraph is invoked twice (once for each partial transition). The following corollary is necessary to show that given a pair of partial transitions, CreateAKGraph will not produce the keys of any node that changed to an intermediate value due to $D \stackrel{T}{\rightarrow} D_{-\triangle T}^{\prime}$, and changed back to its original value due to $D_{-\triangle T}^{\prime} \stackrel{T}{\rightarrow} D^{\prime}$.

Corollary 3.1. Given a relational table $T$, a view graph $G$ which is injective for columns $C$ with respect to $T$, and a database transition $D \stackrel{T}{\rightarrow} D^{\prime}$ with pruned transitional tables $\mathbf{\Delta} T$ and $\boldsymbol{\nabla} T$ :

(a) Let $O_{\triangle}=\operatorname{CreateAKGraph}\left(o_{G}, T, \mathbf{\Delta} T\right)$. $\nexists x, y, z$ $\left(x \in R\left(O_{\triangle}, D^{\prime}\right) \wedge y \in R\left(o_{G}, D\right) \wedge c k v_{o_{G}}(y)=x \wedge\right.$ $\left.v_{C}(y)=v_{C}(z) \wedge z \in R\left(o_{G}, D^{\prime}\right) \wedge c k v_{o_{G}}(z)=x\right)$; and

(b) Let $O_{\nabla}=\operatorname{CreateAKGraph}\left(o_{G_{\text {old }}}, T_{\text {old }}, \boldsymbol{\nabla} T\right)$. $\nexists x, y, z$ $\left(x \in R\left(O_{\nabla}, D^{\prime}\right) \wedge y \in R\left(o_{G}, D\right) \wedge c k v_{o_{G}}(y)=x \wedge\right.$ $\left.v_{C}(y)=v_{C}(z) \wedge z \in R\left(o_{G}, D^{\prime}\right) \wedge c k v_{o_{G}}(z)=x\right)$.

Proof. We prove only case (a), as (b) is analogous.

Suppose that there exist $x, y, z$ contradicting case (a) above. We know from Lemma 3 that there must $\exists w$ such 
that $w \in R\left(o_{G}, D_{-}^{\prime}{ }^{\prime}\right) \wedge c k v_{o_{G}}(w)=x \wedge v_{C}(w) \neq v_{C}(y)$. Let the set $S=\left\{s \in R(T, D) \mid c k v_{o_{G}}(s)=x\right\}$, and similarly, $\bar{S}=\left\{s \in R\left(T, D_{-\mathbf{\Delta} T}^{\prime}\right) \mid c k v_{O_{G}}(s)=x\right\}$. Using Lemma 1 , it follows from $v_{C}(w) \neq v_{C}(y)$ that $S \neq \bar{S}$. Furthermore, from the defintion of injection, we infer from $v_{C}(y)=v_{C}(z)$ that $S^{\prime}=\left\{s \in R\left(T, D^{\prime}\right) \mid c k v_{o_{G}}(s)=x\right\}=S$.

By the definition of pruned transitional tables, $\boldsymbol{\Delta} T \cap \boldsymbol{\nabla} T=\varnothing$, so by Definition 5 it follows from $S=S^{\prime}$ that $S \cap \boldsymbol{\nabla} T=\varnothing$. However, this yields a contradiction: we had previously concluded that $S \neq \bar{S}$, which would require $s \in \boldsymbol{\nabla} T$ for some $s \in S$, and therefore $S \cap \boldsymbol{\nabla} T \neq \varnothing$.

Finally, we can prove that CreateANOpt will not produce spurious updates.

Theorem 3. Given an UPDATE event, a view graph $G$ which is injective for all columns of its top operator, and table $T$, let $G_{\text {affected }}=$ CreateANGraph(UPDATE, $\left.G, T\right)$, and $G_{\text {opt }}=$ CreateANOpt(UPDATE, $\left.G, T\right)$. Then for all valid database transitions $D \stackrel{T}{\rightarrow} D^{\prime}$ with pruned transition tables $\mathbf{\Delta} T$ and $\boldsymbol{\nabla} T$, (OLD_NODE, NEW_NODE) $\in R\left(o_{G_{o p t}}, D^{\prime}\right)$ if and only if (OLD_NODE, NEW_NODE) $\in R\left(o_{G_{\text {affected }}}, D^{\prime}\right)$.

Proof. It is easy to see that the "if" direction is true: from the definition of pruned transitional tables, it follows that pruned transitional tables also satisfy the definition of transitional tables, so the correctness of Theorem 2 is not affected by pruning the transitional tables. The only difference then is that CreateANOpt does not perform the final selection. Since Select can only decrease the cardinality of its input, there will be no affected nodes lost as a result of the change. Thus, (OLD_NODE, NEW_NODE) $\in R\left(o_{G_{\text {affected }}}, D^{\prime}\right)$ implies that (OLD_NODE, NEW_NODE) $\in R\left(o_{G_{\text {opt }}}, D^{\prime}\right)$.

We prove the converse by contradiction. Suppose $\exists$ (OLD_NODE, NEW_NODE) $\in R\left(o_{G_{o p t}}, D^{\prime}\right)$ such that (OLD_NODE, NEW_NODE) $\notin R\left(o_{G_{\text {affected }}}, D^{\prime}\right)$. Since $G_{\text {affected }}=\operatorname{Select}_{(\text {OLD_NODE } \neq \text { NEW_NODE })}\left(G_{\text {opt }}\right)$, it must be the case that OLD_NODE $=$ NEW_NODE. For brevity, we'll refer to this node as $n$.

By line 10, it must be the case that $n \in R\left(O_{\text {old }}\right) \wedge n \in$ $R\left(O_{\text {new }}\right)$. Therefore, by the definition of $O_{\text {new }}$ and $O_{\text {old }}$ (lines 7 and 8), it follows that $x \in R\left(O_{u}, D^{\prime}\right)$ such that $c k v_{o_{G}}(n)=x$. From the definition of $O_{u}$ (line 6), we see that either (a) $x \in R\left(O_{\triangle} k e y\right)$ or (b) $x \in R\left(O_{\nabla} k e y\right)$. However, this yields a contradiction: in case (a) this violates Corollary 3.1(a), and in case (b) it violates Corollary 3.1(b). Therefore, our initial assumption that $n \in R\left(o_{G_{o p t}}, D^{\prime}\right)$ must have been false.

\section{F.4. Additional Optimizations}

In the previous section, we showed that for injective view graphs, we can avoid performing an expensive tagger- level comparison of OLD_NODE and NEW_NODE for UPDATE events. If a view is not injective, then we generally need to keep this selection condition. In certain cases, however, we can still optimize the performance by pushing down the selection condition to the relational level.

If a view graph $G$ is injective except for the presence of a non-injective aggregate functions (e.g. min, max, count, etc.) in GroupBy operators, such as in the example shown in Appendix E.1, then it is not necessary to compare the entire old and new XML nodes. Instead, it is only necessary to compare the values of these aggregates. Since this is a comparison of numeric values with no nesting involved, it can be pushed down to the relational engine, thus avoiding the need to perform an expensive string comparison in the tagger.

This is just one of many possible optimizations to avoid performing a tagger-level comparison for non-injective views. A possible direction of future work is to identify the general class of views where the top-level selection can be pushed down to the relational level.

\section{G. Additional Experimental Results}

In this section, we show the experimental results of varying the parameter leaf tuples per XML element, number of leaf tuples, and number of satisfied triggers. In all experiments, we vary the parameter of interest and use default values for the rest. Note that varying these three parameters, unlike the two parameters evaluated in Section 6, changes the number of tuples inserted into the temporary table by the SQL trigger action. This introduces additional overhead not directly relevant to trigger processing. To isolate this effect, we first compute all the rows produced by the trigger, but only insert the maximum row into the temporary table (by using the max aggregate function), thus keeping the cost of the relational inserts constant.



Figure 22. Varying the fanout. 


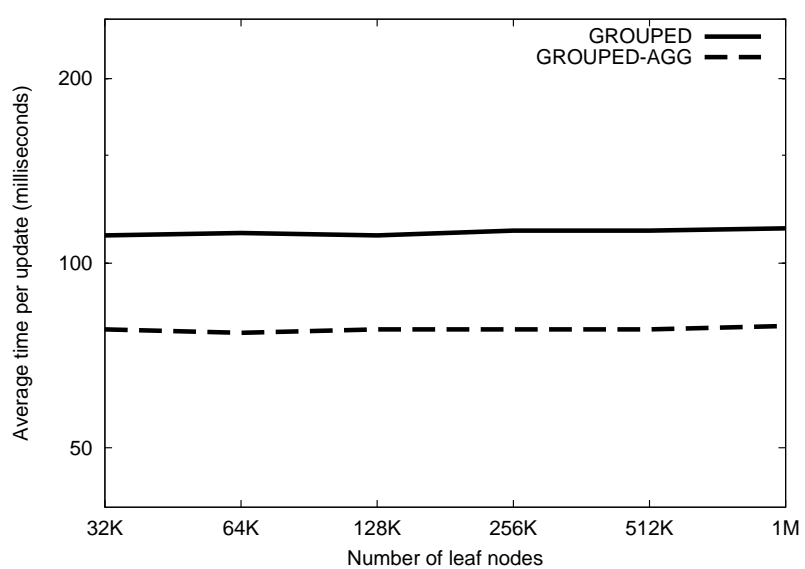

Figure 23. Varying the data size.

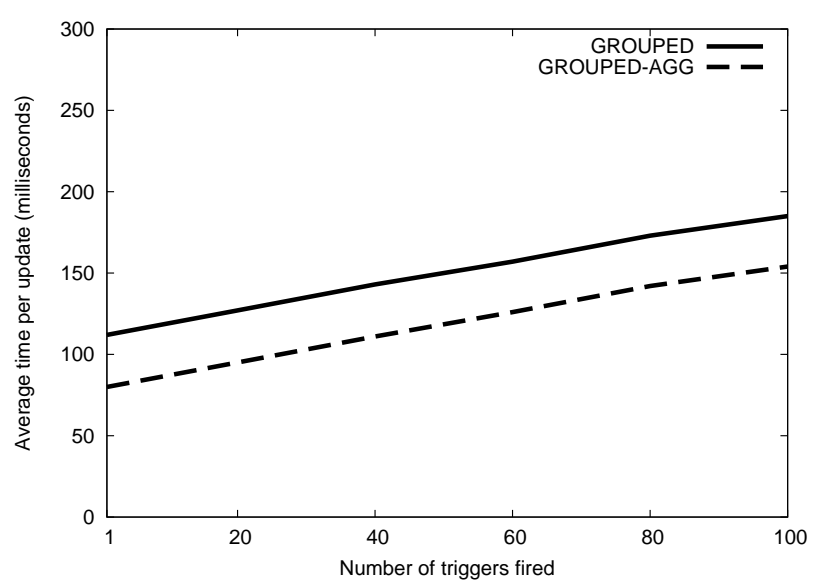

Figure 24. Varying the number of fired triggers.

\section{G.1. Varying Leaf Tuples per XML Element}

Figure 22 shows the effect of varying the fanout, the number of leaf tuples per XML element. GROUPED and GROUPED-AGG have the same relative performance, and there is only a small increase in runtime as the fanout increases. This increase is due primarily to the fact that the OuterUnion intermediate result grows as OLD_NODE and NEW_NODE become larger.

\section{G.2. Varying Number of Leaf Tuples}

We vary the data size by varying the number of leaf tuples; the result is shown in Figure 23. GROUPED and GROUPED-AGG scale gracefully when the data size increases. This is because, although the total number of leaf tuples increases, the number of leaf nodes in the affected XML element remains the same. This graph shows that our system indeed benefits from not materializing the entire XML view, so that we only need to compute a small fraction of leaf nodes.

\section{G.3. Varying Number of Satisfied Triggers}

Figure 24 shows the effect of varying the number of satisfied triggers. GROUPED and GROUPED-AGG have the same relative performance and their runtime linearly increases with the number of satisfied triggers. This is because the number of computed (OLD_NODE, NEW_NODE) pairs increases with the number of satisfied triggers. 